Received: 13 April 2018

Accepted: 30 July 2018

Published online: 13 August 2018

SCIENTIFIC REP

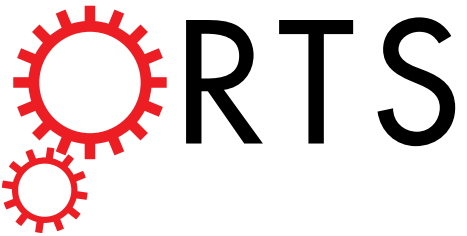

\title{
OPEN
}

\section{The Gpr1-regulated Sur7 family protein Sfp2 is required for hyphal growth and cell wall stability in the mycoparasite Trichoderma atroviride}

\author{
Lea Atanasova ${ }^{1,6}$, Sabine Gruber ${ }^{1}$, Alexander Lichius ${ }^{1}$, Theresa Radebner ${ }^{2}$, \\ Leoni Abendstein ${ }^{1}$, Martin Münsterkötter $\mathbb{1}^{3,4}{ }^{3}$, Nancy Stralis-Pavese ${ }^{5}$, Paweł P. Łabaj ${ }^{5}$, \\ David P. Kreil ${ }^{5}$ \& Susanne Zeilinger ${ }^{1,2}$
}

Mycoparasites, e.g. fungi feeding on other fungi, are prominent within the genus Trichoderma and represent a promising alternative to chemical fungicides for plant disease control. We previously showed that the seven-transmembrane receptor Gpr1 regulates mycelial growth and asexual development and governs mycoparasitism-related processes in Trichoderma atroviride. We now describe the identification of genes being targeted by Gpr1 under mycoparasitic conditions. The identified gene set includes a candidate, sfp2, encoding a protein of the fungal-specific Sur7 superfamily, whose upregulation in $T$. atroviride upon interaction with a fungal prey is dependent on Gpr1. Sur7 family proteins are typical residents of membrane microdomains such as the membrane compartment of Can1 (MCC)/eisosome in yeast. We found that GFP-labeled Gpr1 and Sfp2 proteins show partly overlapping localization patterns in $T$. atroviride hyphae, which may point to shared functions and potential interaction during signal perception and endocytosis. Deletion of $s f p 2$ caused heavily altered colony morphology, defects in polarized growth, cell wall integrity and endocytosis, and significantly reduced mycoparasitic activity, whereas $s f p 2$ overexpression enhanced full overgrowth and killing of the prey. Transcriptional activation of a chitinase specific for hyphal growth and network formation and strong downregulation of chitin synthase-encoding genes were observed in $\Delta s f p 2$. Taken together, these findings imply crucial functions of Sfp2 in hyphal morphogenesis of $T$. atroviride and its interaction with prey fungi.

Fungal pathogens causing plant disease and thereby losses in natural resources pose a common problem in agriculture. The abusive application of chemical fungicides for plant disease control often renders plant-pathogenic fungi resistant and leads to a detrimental pollution of the environment ${ }^{1}$. A sustainable alternative to chemical fungicides is the use of antagonistic microorganisms ${ }^{2}$. Members of the genus Trichoderma (teleomorph Hypocrea, Ascomycota) are superior mycoparasites - fungi that can parasitize and kill other fungi - rendering these species potent biocontrol agents for plant disease control ${ }^{3}$.

Trichoderma spp. may recognize plant-pathogenic prey fungi via small molecules that are released by the pathogen already before contact. These molecules are supposed to bind Trichoderma receptors such as seven-transmembrane G protein-coupled receptors (GPCRs), thereby eliciting a signaling cascade that triggers

${ }^{1}$ Institute of Microbiology, University of Innsbruck, Innsbruck, Austria. ${ }^{2}$ Institute of Chemical, Environmental \& Bioscience Engineering, TU Wien, Vienna, Austria. ${ }^{3}$ MIPS - Institute of Bioinformatics and Systems Biology, Helmholtz Zentrum München, Munich, Germany. ${ }^{4}$ Functional Genomics and Bioinformatics, Sopron University, Sopron, Hungary. ${ }^{5}$ Chair of Bioinformatics, Boku University Vienna, Vienna, Austria. ${ }^{6}$ Present address: Institute of Food Technology, University of Natural Resources and Life Sciences (BOKU), Vienna, Austria. Sabine Gruber and Alexander Lichius contributed equally to this work. Correspondence and requests for materials should be addressed to S.Z. (email: Susanne.zeilinger@uibk.ac.at) 
downstream responses ${ }^{4}$. The mycoparasitic response includes enhancement of the expression of genes that encode enzymes for the biosynthesis of secondary metabolites and for cell wall lysis ${ }^{5}$.

In filamentous fungi, GPCRs sense pheromones, sugars, amino acids, nitrogen sources and even photons ${ }^{6}$. More than 50 putative GPCRs have been identified in the genome of the mycoparasite Trichoderma atroviride and four of these genes ( $g p r 1, g p r 2, g p r 3$ and $g p r 4)$ were previously isolated and characterized ${ }^{7,8}$. Gpr1 turned out to play an important role in the antagonistic interactions of T. atroviride with prey fungi by governing mycoparasitism-related processes. $g$ prl-silenced mutants were avirulent in confrontation assays due to their inability to attach to and lyse prey hyphae?.

Micro- or nanodomain structures of the plasma membrane are supposed to act as organizing centers for dynamic processes such as membrane transport, polarized growth, and signal transduction by influencing the organization and dynamic association of receptors with interacting proteins ${ }^{10}$. The Saccharomyces cerevisiae plasma membrane contains different types of subdomains exhibiting a composition, structure, and biological function distinct from the surrounding membrane $\mathrm{e}^{11-13}$. One of them is a protein-organized microdomain known as Membrane Compartment occupied by Can1 (MCC), which corresponds to inward furrows on the plasma membrane $e^{11,14,15}$. Sur7 family proteins are typical residents of such membrane microdomains that are stabilized by a complex of cytosolic proteins termed the eisosome $\mathrm{e}^{14,16}$. The eisosomal portion of proteins assemble into filaments and curve the membrane to form the furrows ${ }^{15}$. Amongst others, two families of tetraspan proteins, one comprising Sur7 and its paralogs Fmp45, Pun1, and Ynl194c (Sur7 protein family), and the other including Nce102 and Fhn1, were discovered in the MCC $^{17-19}$. Sur7-containing MCC domains are important for plasma membrane organization, sphingolipid homeostasis, and cell wall morphogenesis (for review see ${ }^{15}$ ). While S. cerevisiae SUR7 deletion mutants showed reduced sporulation but had no obvious macroscopic growth phenotype $^{14,20}$, CaSur7 of the opportunistic human pathogen Candida albicans promoted proper spatial organization of cell wall synthesis and plasma membrane organization as well avirulence ${ }^{17,21}$, and the mutants showed a clear growth phenotype resembling the one caused by the inhibition of $\beta$-glucan synthesis ${ }^{22}$. In the filamentous fungus Aspergillus nidulans, deletion of surG (sur7 orthologue) did not lead to any obvious growth phenotypes ${ }^{23}$. Nevertheless, the yeast Sur7 paralogs Fmp45, Pun1, and YNL194C affect the response to nitrogen stress, cell wall integrity, and survival in stationary phase $\mathrm{i}^{15,24,25}$.

Here, we describe the functional characterization of the T. atroviride $s f p 2$ gene encoding a Sur7 family protein that emerged from transcriptomics studies as being regulated by the Gprl receptor under mycoparasitic conditions. Phylogenetic analysis further revealed Sfp2 as an orthologue of yeast Pun1, a membrane protein that until now has not been studied in filamentous fungi. We identified Sfp2 as an important player in T. atroviride that affects hyphal growth and mycelial network formation, cell wall remodeling and stability, endocytosis, osmotic stress resistance, as well as mycoparasitism.

\section{Results}

Identification of prey-regulated genes whose transcription is affected by Gpr1. Prey-regulated genes transcriptionally affected by $g$ pr 1 silencing were identified by microarray-based genome-wide comparison of gene expression in T. atroviride strain P1 (wild type; WT) and its $g$ pr1-silenced mutant (gpr1-si) in response to interaction with the plant pathogenic prey fungus Rhizoctonia solani. A putative group of genes affected by Gpr 1 during mycoparasitism emerged, with 645 genes being differently regulated in the response to the prey fungus in the gpr1-si mutant compared to the wild type strain (Table S1). Of these differently regulated genes, $80 \%$ encoded proteins with significant similarity to well-characterized fungal proteins or at least contained protein domains with known functions. About $60 \%$ showed a higher-expression response in the WT than in the mutant, i.e. were either upregulated instead of downregulated in the prey response, or were more upregulated, or were less downregulated. Of these, about $7 \%$ were coding for secreted proteins, whereas from the genes with a lower-expression response in the WT compared to the mutant, about $10 \%$ were coding for secreted proteins.

An analysis of assigned functional categories (FunCat) suggested that the most robustly over-represented processes characterizing genes that exhibited a prey response dependent on the Gpr1 receptor included specific fungal information pathways (Table S2). Specifically we found support for translation (FunCat ID 12.04), protein modification (14.07; strong support), protein targeting, sorting and translocation (14.04; strong), and protein activity regulation (18.01; strong). Further implicated categories included RNA synthesis (12.01; strong), genes involved in cellular transport (ER to Golgi transport 20.09.07.03; vesicle formation 20.09.07.25; intra Golgi transport 20.09.07.05; all strong) and metabolism such as phosphate metabolism (01.04; with moderately strong support), sugar, glucoside, polyol and carboxylate anabolism (01.05.02.04; moderate), and peptide antibiotics metabolism (01.20.37.03; supported by some analyses). All of these were implicated as over-represented amongst the genes dependent on Gpr1 in the response of T. atroviride to a fungal prey.

Amongst the genes showing a higher-expression response in the WT than in the mutant were mycoparasitism-related candidates, such as genes encoding aspartyl proteases; GprK- and PTH11-type GPCRs; non-ribosomal peptide synthases (NRPS) including the peptaibol synthetase Tex1; several predicted SSCRPs (including putative hydrophobins and a C-type lectin); GH18 chitinases; a putative GH75 chitosanase; a protein with a Carbohydrate-Binding Module (CBM) also known as LysM domain belonging to Family 50 (putative Tal1); glutathione S-transferases and a glutathione synthetase, the osmosensing-associated Tmk3 MAP kinase, and S-adenosyl methyltransferases, most prominent the methyltransferase Lae1, a key regulator of asexual development and mycoparasitism in T. atroviride ${ }^{26}$. In addition, we found genes involved in metabolism (encoding putative $\alpha$-1,2-mannosidase (GH92), $\beta$-mannosidase (GH2), amidase, 1,4- $\alpha$-glucan branching enzyme, members of GH63 and GH3), several GTP-binding and GTPase activating proteins, several subunits of the SWI-SNF chromatin remodeling complex, an orthologue of the T. reesei zink finger repressor of cellulase and xylanase expression ace1, as well as the blu7 transcription factor gene involved in light response ${ }^{27}$, amongst the genes showing a Gpr1-dependent response (Table S1). 


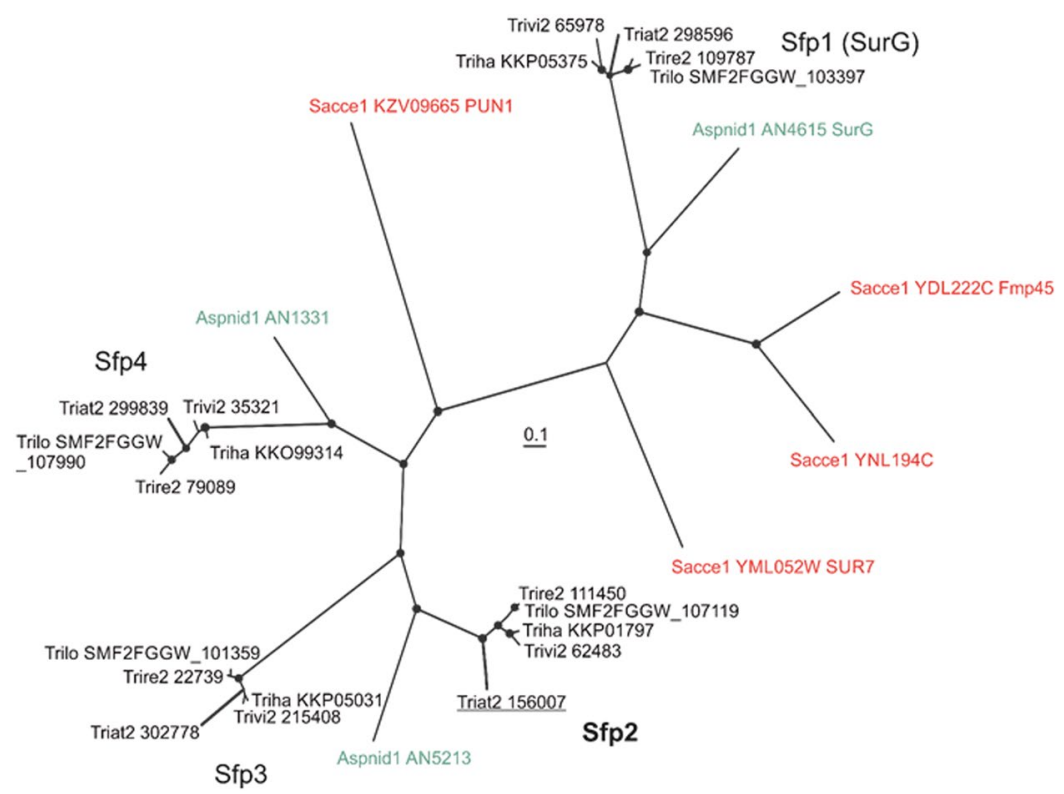

Figure 1. Bayesian phylogram based on amino acid sequences from Sur7 orthologues of S. cerevisiae, A. nidulans and Trichoderma spp. The Bayesian analysis ran for three million MCMC generations and a strict consensus tree was obtained using a Dayhoff amino acid substitution model. PP values lower than 0.95 were not considered significant and are not shown on the resulting phylogram. Sacce1, Aspnid1, Triat2, Trire2, Trilo SMF2FGGW, Trivi2 and Triha are derived from the respective available genome versions of S. cerevisiae, A. nidulans FGSC A4, T. atroviride IMI 206040, T. reesei QM 6a, T. longibrachiatum SMF2, T. virens Gv29-8 and T. harzianum T6776, respectively. The underlined sequence represents the T. atroviride Sur7 family protein Sfp2.

In total, 99 genes with a Gpr1-dependent response encoded transmembrane (TM) proteins with one to sixteen transmembrane domains. These included GprK- and PTH11-type GPCRs, iron permease proteins, as well as RTA1-domain containing proteins (a lipid-translocating exporter family in S. cerevisiae). Most of the identified candidates with four transmembrane domains were predicted to be involved in vesicle-mediated transport such as a Sur7 family protein and Sft2-like vesicle transport proteins.

The genomic environment of gpr1. We were interested in identifying genes whose close proximity to gprl on the chromosome may indicate (co)regulation or transcriptional interference. Comparative analysis of the contig region around gprl orthologues in T. reesei $(\operatorname{Tr} 123806)$, T. virens (Tv33049), and T. atroviride (Ta160995) genomes revealed a strongly conserved genetic structure in the three Trichoderma species (Fig. S1). However, in T. virens the respective genes were transcribed in the opposite direction compared to T. atroviride and T. reesei, indicating that a scaffold inversion had occurred during evolution.

Examining the syntenic region between bp 172758 and 259517 on T. atroviride contig 29 revealed genes encoding peptidases, MFS transporters, a sulphatase, the eliciting plant response-like protein Epl $3^{28}$, a fungal-specific transcription factor, and a member of the fungal-specific Sur7 family (Fig. S1). A search within the gene set being differently regulated in the prey responses of the T. atroviride wild type and the gprl-si mutant revealed that the transcription of the gene encoding the Sur7 family protein (further designated Sur7-family protein $2, s f p 2$ ) is dependent on Gpr1. The observed sfp2 (Ta156007) mRNA levels were specifically upregulated upon prey contact in the wild type but not in the gpr1-si mutant (Table S1).

The $s f p 2$ open reading frame consists of three exons and two introns and encodes a protein of 318 amino acids with four predicted transmembrane domains and a fungal-specific Sur7 domain (pfam06687). Phylogenetic analysis of Sur7 family proteins from S. cerevisiae, A. nidulans and different Trichoderma species revealed four clades comprising Trichoderma Sur7 family members with one clade representing orthologues of $A$. nidulans SurG (Fig. 1). SurG is a true orthologue of S. cerevisiae Sur $7^{23}$ for which the functions were already reported. According to the phylogram (Fig. 1), the S. cerevisiae Sur7 paralog Pun1 seems to be the common ancestor of the second supported clade, which further comprises three supported subclades. T. atroviride Sfp 2 and its paralogs from other Trichoderma species hence are more closely related to yeast Pun1 than to Sur7.

Sfp2 impacts hyphal growth and branching. To assess the biological role of Sfp2 in T. atroviride, $s f p 2$ gene deletion mutants were generated. Although all of the resulting 25 transformants showed hygromycin B-resistance, further analysis yielded only one mitotically stable mutant with homologous integration and hence deletion of the $s f p 2$ open reading frame. Complemented strains (REsfp2) were generated by reintroducing $s f p 2$ under control of the constitutive pki1 promoter (Ppki1::sfp2-mEGFP construct) into a random site of the genome of the $\Delta s f p 2$ mutant. Strains overexpressing $s f p 2$ (OEsfp2) were obtained by introducing P $p k i 1:: s f p 2$ with and without C-terminal mEGFP tag into the WT background. As sfp2 expression turned out to be governed by Gpr1, 


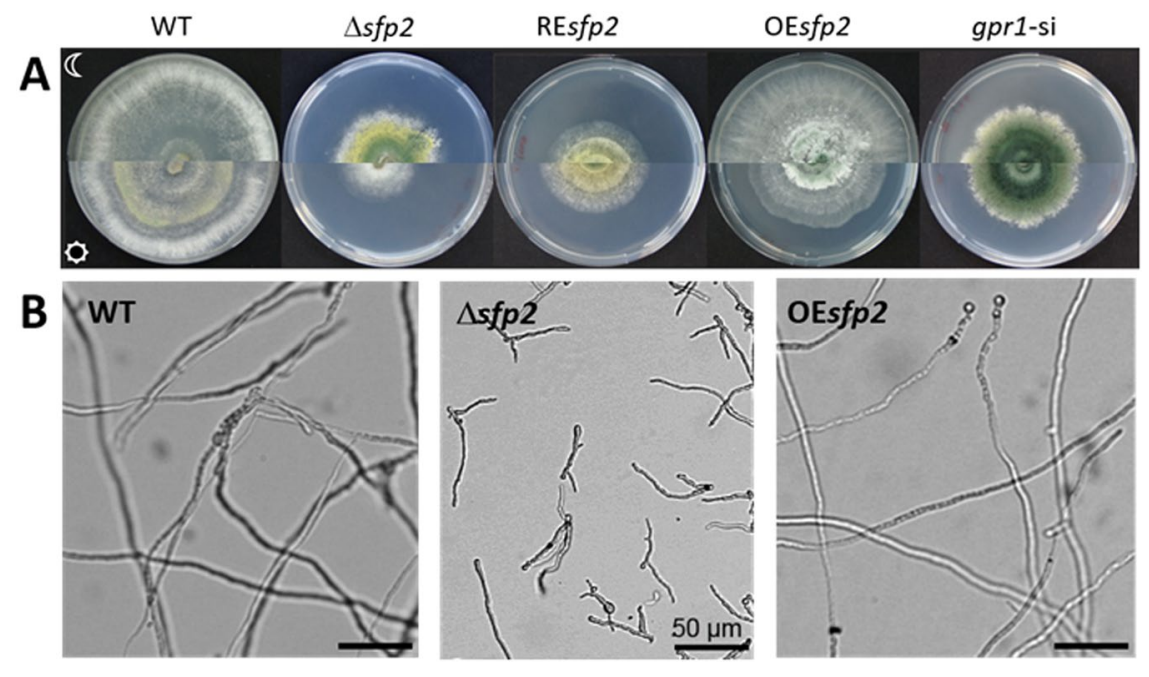

Figure 2. Phenotypical characterization of T. atroviride sfp2 and gpr 1 mutants. (A) Strains grown for five days on PDA plates in complete darkness (upper part of the plates) or under cycling daylight (lower part of the plates). (B) Microscopic images of hyphae from T. atroviride WT, $s f p 2$ deletion $(\Delta s f p 2)$ and overexpression (OEsfp2) mutants originating from conidia grown in PDB for $24 \mathrm{~h}$. Differences in hyphal branching and germ tube lengths were observed between the strains with $\Delta s f p 2$ exhibiting shorter and thinner germ tubes than the WT and the OEsfp2 strain.

the $g \operatorname{pr} 1$-silenced mutant $g \operatorname{pr} 1$ sil- $8^{9}$ was included in the study to identify putative overlapping phenotypes resulting from $s f p 2$ knock-out and $g p r 1$ knock-down, respectively.

Striking differences in macro- and micro-morphology between the WT and the tested mutants were observed upon cultivation on complex medium agar plates in complete darkness as well as under cycling illumination. The $\Delta s f p 2$ mutant, similar to gpr1 sil-8, formed compact colonies with significantly less aerial hyphae than the WT, but instead exhibited invasive growth with hyphae entering the solid medium. In T. atroviride, conidia production is light-dependent ${ }^{29}$. Accordingly, the WT did not conidiate when cultivated in complete darkness. In contrast, $\Delta s f p 2$ as well as $g p r 1$ sil-8, showed permanent, light-independent conidiation (Fig. $2 \mathrm{~A}$ ). The heavily reduced growth of the $\Delta s f p 2$ mutant in light was partially restored by re-introducing the $s f p 2$ gene and the re-transformed strain still showed slight conidiation in darkness. Growth of the $s f p 2$ mutant strains was also monitored in liquid culture by incubating the WT and the two mutant strains, $\Delta s f p 2$ and OEsfp2, in PDB for $12 \mathrm{~h}$. Under these conditions, the $\Delta s f p 2$ mutant displayed retarded germination and restricted hyphal elongation compared to the WT and the OEsfp2 strain. After $12 \mathrm{~h}$ in PDB, $100 \%$ of the WT, $85 \%$ of OEsfp 2 but only $28 \%$ of the $\Delta s f p 2$ conidia had germinated, all with unipolar germ tubes. Similar results were obtained with synthetic minimal medium. Microscopic examination of hyphae after prolonged cultivation $(24 \mathrm{~h})$ revealed abnormal, enhanced branching of $\Delta s f p 2$ indicating a polarity defect caused by deletion of the $s f p 2$ gene (Fig. 2B).

Sfp2 is involved in osmotic stress regulation and mycoparasitic activity. Our previous analyses of gpr1-silenced mutants revealed a complete loss of mycoparasitic activity, i.e. inability of the mutants to attach to, overgrow and lyse prey hyphae ${ }^{9}$. To test whether $s f p 2$ is involved in mycoparasitism, plate confrontation assays against R. solani as fungal prey were performed. Compared to the WT that attacked and overgrew the prey within seven days, the $\Delta s f p 2$ mutant had a reduced antagonistic potential evidenced by a lower growth inhibition of $R$. solani. The ability to suppress the growth of the fungal prey slightly recovered in the genetically complemented Resfp2 mutant, while overexpression of $s f p 2$ led to a very strong mycoparasitic response with enhanced overgrowth and killing of the prey (Fig. 3A).

Exposure to sorbitol-mediated osmotic stress and sodium chloride-mediated salt stress resulted in strongly impaired growth of $\Delta s f p 2$ (Fig. 3B). Interestingly, the gpr1-si mutant tolerated osmotic stress better than $\Delta s f p 2$, but was strongly susceptible to salt stress. Both, salt as well as osmotic stress, however, only slightly altered the growth rate of OEsfp2 and the WT (Fig. 3C,D).

Sfp2 is required for cell wall stability and endocytosis. To test cell wall stability, strains were grown on PDA supplemented with $200 \mu \mathrm{M}$ Congo Red (CR), a dye that forms a complex with (helical) chain parts of chitin networks and results in a loss of cell wall rigidity due to compromised lateral interaction between the helices ${ }^{30}$. CR strongly affected $\Delta s f p 2$ causing a $43 \%$ growth reduction compared to the control condition (PDA only), whereas growth of the WT and the gpr1-si mutant was not significantly altered by the addition of the dye (Fig. 3B).

We next tested the expression of genes putatively involved in the remodeling, repair and degradation of the fungal cell wall, such as chitinases and chitin synthases, when T. atroviride was confronted with itself (control) or R. solani (mycoparasitism). Subgroup C chitinases encoded by tac2 and tac6 are responsible for hyphal growth and network formation of T. atroviride but not for mycoparasitism ${ }^{31,32}$. In our study, transcription of tac 2 and tac6 was strongly downregulated in the WT but also in $\Delta s f p 2$ when confronted with $R$. solani. Upon contact with its own hyphae, however, $\Delta s f p 2$ induced the expression of tac 2 more than 15 fold (Fig. 4 ). These results indicate that 

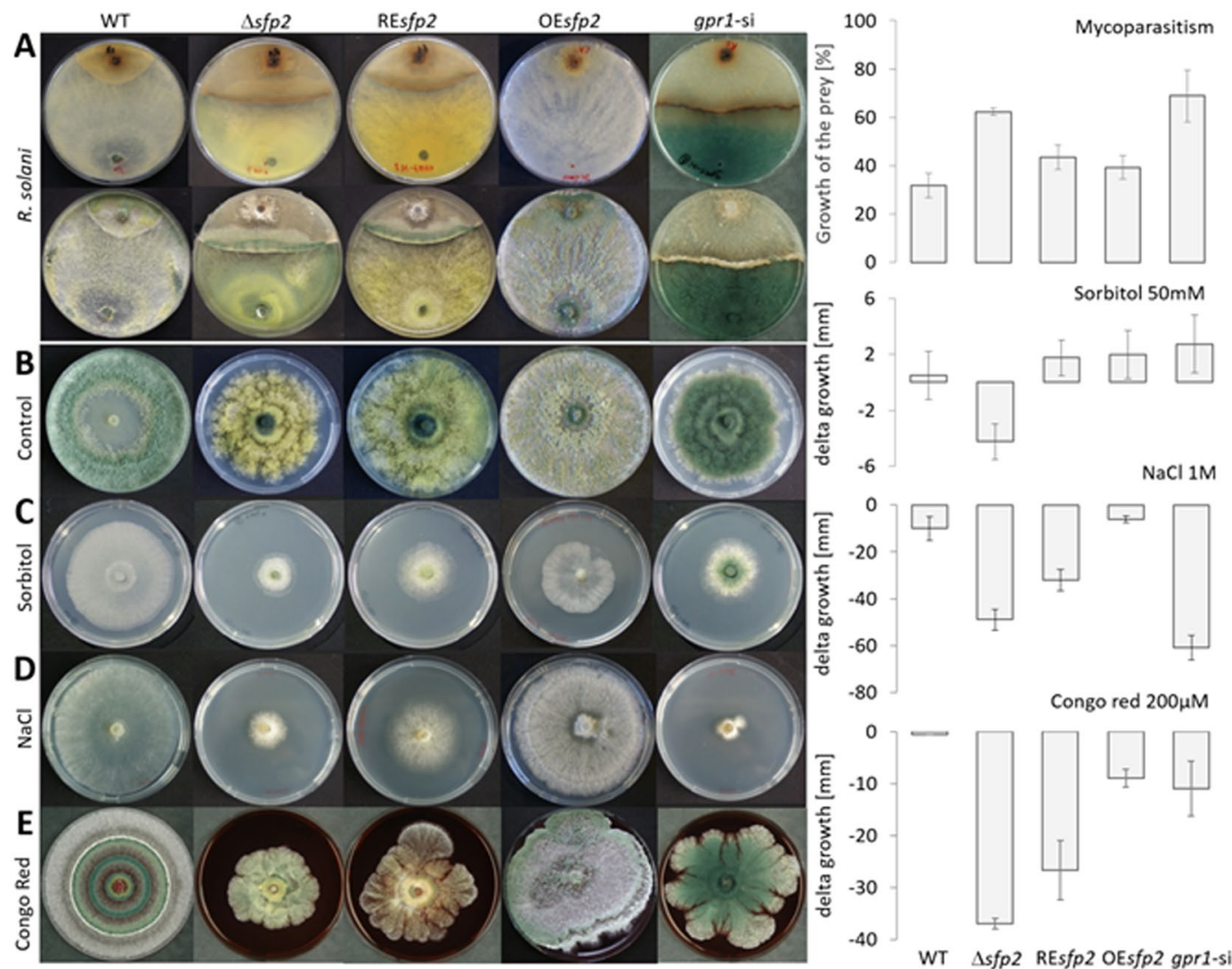

Figure 3. Involvement of Sfp 2 in antagonism of T. atroviride against $R$. solani and in the response to salt, osmotic and cell wall stress. (A) Dual confrontation assays against $R$. solani (back and front sides of the plates are shown). (B) Cultures of T. atroviride strains on PDA without any stressor. Growth on PDA supplemented with $50 \mathrm{mM}$ sorbitol (C), $1 \mathrm{M} \mathrm{NaCl}(\mathbf{D})$ and $200 \mu \mathrm{M}$ Congo Red (E). Plates were incubated in darkness at $25^{\circ} \mathrm{C}$ for seven days. Graphs represent the antagonistic potential and stress sensitivity of the different T. atroviride strains. Error bars annotate the standard deviations based on at least three biological replicates.

$s f p 2$ might regulate the machinery for self-cell-wall hydrolysis and thereby control growth and network formation of T. atroviride hyphae. Elevated tac 2 transcription levels in the $\Delta s f p 2$ mutant could be due to its increased hyphal branching (Fig. 2B). However, when grown in shaking liquid medium (reduced self-contact) the expression of tac2 was reduced in $\Delta s f p 2$ compared to the WT. The addition of CR into the liquid medium, however, induced tac2 transcription in $\Delta s f p 2$ and led to reduced tac2 mRNA levels in OEsfp 2 compared to the WT and compared to growth without CR (Fig. S2). We assume that the overexpression of $s f p 2$ likely causes increased cell wall stability that better endures the loss of rigidity due to cell wall intercalation and consequently reduces expression of tac 2 . Surprisingly, the expression of tac 6 was not affected in $s f p 2$ mutants during self-confrontation. Transcription of ech42 (encoding endochitinase 42 that is the most abundant Trichoderma chitinase) was massively induced in $\Delta s f p 2$, particularly when confronted with itself but also in contact with $R$. solani. In liquid cultures, ech 42 was constitutively expressed in all $s f p 2$ mutant strains and the WT.

Chitin synthases (CHS) are essential for hyphal growth and are supposed to be involved in the repair of the fungal cell wall ${ }^{33-35}$. Decreased transcriptional levels of two chitin synthase-encoding genes, chs 1 (encoding a CHS with a myosin domain) and chs2 (encoding a class I CHS), were evident in $\Delta s f p 2$ upon self-confrontation (Fig. 4). However, upon confrontation with $R$. solani transcription of both chitin synthase genes was significantly upregulated in the $\Delta s f p 2$ mutant (up to 4-fold), whereas it was downregulated in the WT and in the $s f p 2$ overexpression mutant compared to non-antagonistic growth conditions. In addition, actin, that plays an important role in forming microfilaments ${ }^{36}$, was transcriptionally upregulated in $\Delta s f p 2$ upon self-contact, whereas in OEsfp2 its transcription was significantly downregulated relative to the WT (Fig. 4). These results indicate that cell wall integrity, and consequently cell wall synthesis and autolysis, are likely dependent on Sfp2. This is reflected in the $\Delta s f p 2$ growth phenotype that lacks hyphal diversification, i.e. all hyphae in the periphery and subperiphery of the colony looked similar and did not differentiate into leading hyphae and primary and secondary lateral branches. We further tested this hypothesis by staining the cell wall with various dyes selective for $\beta$-1,4-glucans, including chitin, thereby revealing some additional features of the $\Delta s f p 2$ hyphal growth phenotype (Fig. 5). Hyphae of $\Delta s f p 2$ showed a pronounced deposition of chitin at tip apices compared to the WT and furthermore, displayed increased sensitivity to $\mathrm{CR}$ which led to extensive isotropic tip swelling (arrows and arrowheads in Fig. 5A). In addition, the average distance between septa $\left(\Delta s f p 2=26.0 \mu \mathrm{m}, \mathrm{WT}=85 \mu \mathrm{m} ; \mathrm{n}=60\right.$; ANOVA $\left.\operatorname{Pr}<2^{-16}\right)$ and the average hyphal diameter $\left(\Delta s f p 2=5.6 \mu \mathrm{m}\right.$, WT $=12.6 \mu \mathrm{m} ; \mathrm{n}=100$; ANOVA $\left.\operatorname{Pr}<2^{-16}\right)$ in the mutant were 

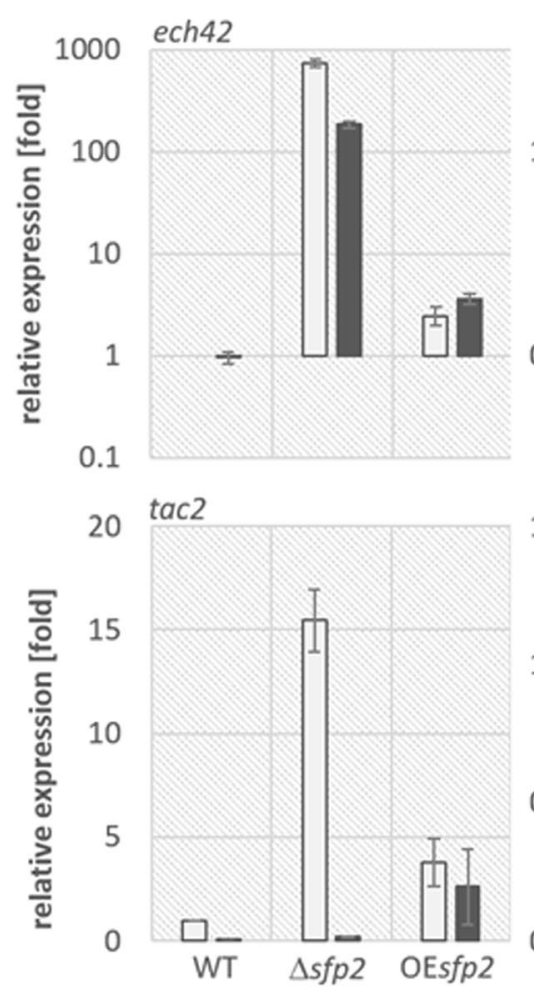
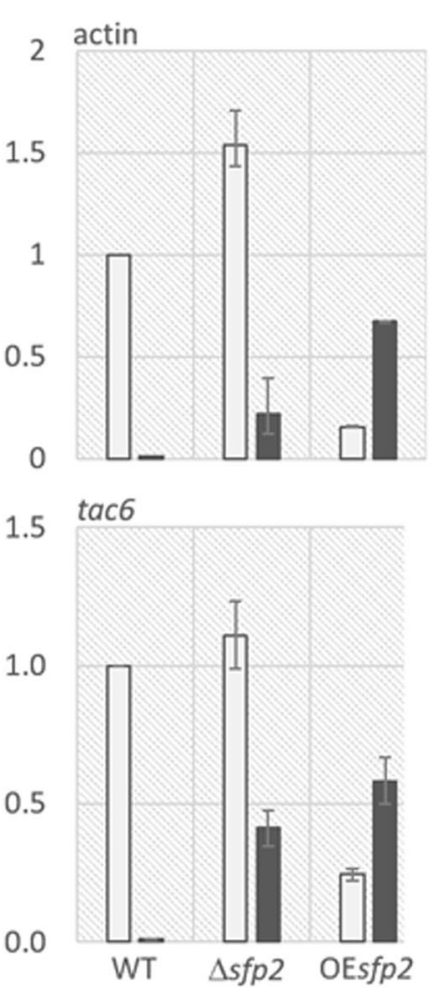

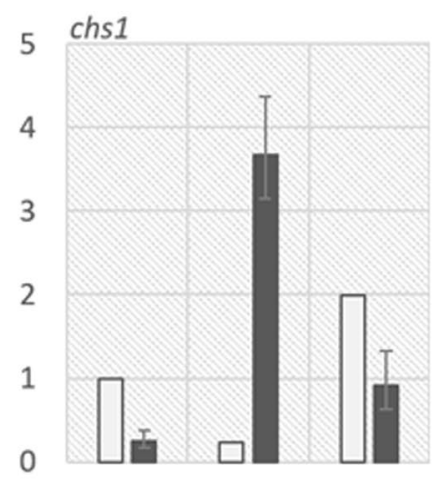

5

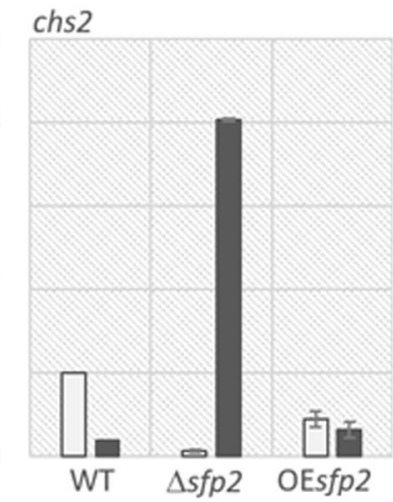

Figure 4. Transcriptional analysis of chitinase, actin and chitin synthase genes in T. atroviride during selfconfrontation (white bars) and confrontation with R. solani (black bars, sampling at the hyphal contact stage) in WT, sfp2 deletion and overexpression mutants. Transcript patterns of sgC chitinase genes tac2 and tac6, chitinase $e c h 42^{31}$, two chitin synthases, chs 1 (Ta142365) with myosin domain and class I chs2 (Ta323101), as well as actin gene (Ta297070) were analyzed to elucidate the effect of $s f p 2$ deletion on the regulation of cell wall remodeling activities. sar 1 was used as a reference gene $\mathrm{e}^{7}$. The error bars represent standard deviation based on at least three sample replicates.

significantly reduced when compared to the WT (Fig. 5A,B). Quantification of the ratio of the relative dye fluorescence between tip apex and subapex revealed interesting changes in the cell wall deposition pattern between $\Delta s f p 2$ and the WT (Fig. 5C-E). In comparison to the WT, the absolute amount of CFW and SPF dye incorporated at the tip apices of the mutant was elevated (CR was equal), whereas the signal intensity of all three dyes in the subapex was much reduced. Consequently, the ratio between apical and subapical fluorescence of all dyes in the mutant was significantly higher compared to the WT. This indicates pronounced cell wall deposition at growing hyphal tips but weaker consolidation of cell wall structure in the subapex of $\Delta s f p 2$ hyphae. Together, these features support the notion of a highly disturbed and chemically altered cell wall organization in the $\Delta s f p 2$ mutant, leading to a fundamentally de-regulated control over hyphal morphogenesis. Another hint for this might be a possible preference of two distinct septal distances of about 15 and $30 \mu \mathrm{m}$ in $\Delta s f p 2$ that can be observed as slightly bimodal shape of the violin plot of $\Delta s f p 2$ septal distance (Fig. 5B).

Sur7 family proteins are typical residents of membrane microdomains such as the MCC/eisosome in yeasts ${ }^{15}$. We therefore tested the effect of Sfp2 on endocytosis using the membrane-selective FM4-64 dye as marker for endosome internalization (Fig. 6). While OEsfp2 showed slightly accelerated dye uptake compared to the WT, $\Delta s f p 2$ displayed significantly delayed endocytosis, obvious by the first appearance of intracellular dye molecules only after 45-60 min post FM4-64 addition. An equivalent staining pattern occurred in the WT already after $10-15 \mathrm{~min}$.

Gpr1 and Sfp2 show overlapping but not identical localization patterns. Life-cell imaging analyses of T. atroviride transformants expressing Gpr1-mEGFP and Sfp2-mEGFP, respectively, showed that both proteins have similar, greatly overlapping but not fully identical, subcellular localization patterns (Fig. 7). As expected, Gpr1 and Sfp2 both reside at the plasma membrane, including highly pronounced accumulation at septa, and are associated to intracellular membrane clusters and vesicles, presumably associated to the endocytic pathway. Both proteins furthermore localize to what appear to be tubular vacuoles in the subapical compartment of leading hyphae. Most notably, Sfp2 is - in contrast to Gpr1 - also part of the Spitzenkörper (arrows in Fig. 7B,C) and clusters at the position of the subapical endocytic ring (arrowheads in Fig. 7B). These subcellular localizations strongly suggest that Sfp2 is part of the exo-/endocytic machinery driving polarized tip growth, which is in line with the observed endocytosis defect of $\Delta s f p 2$. 

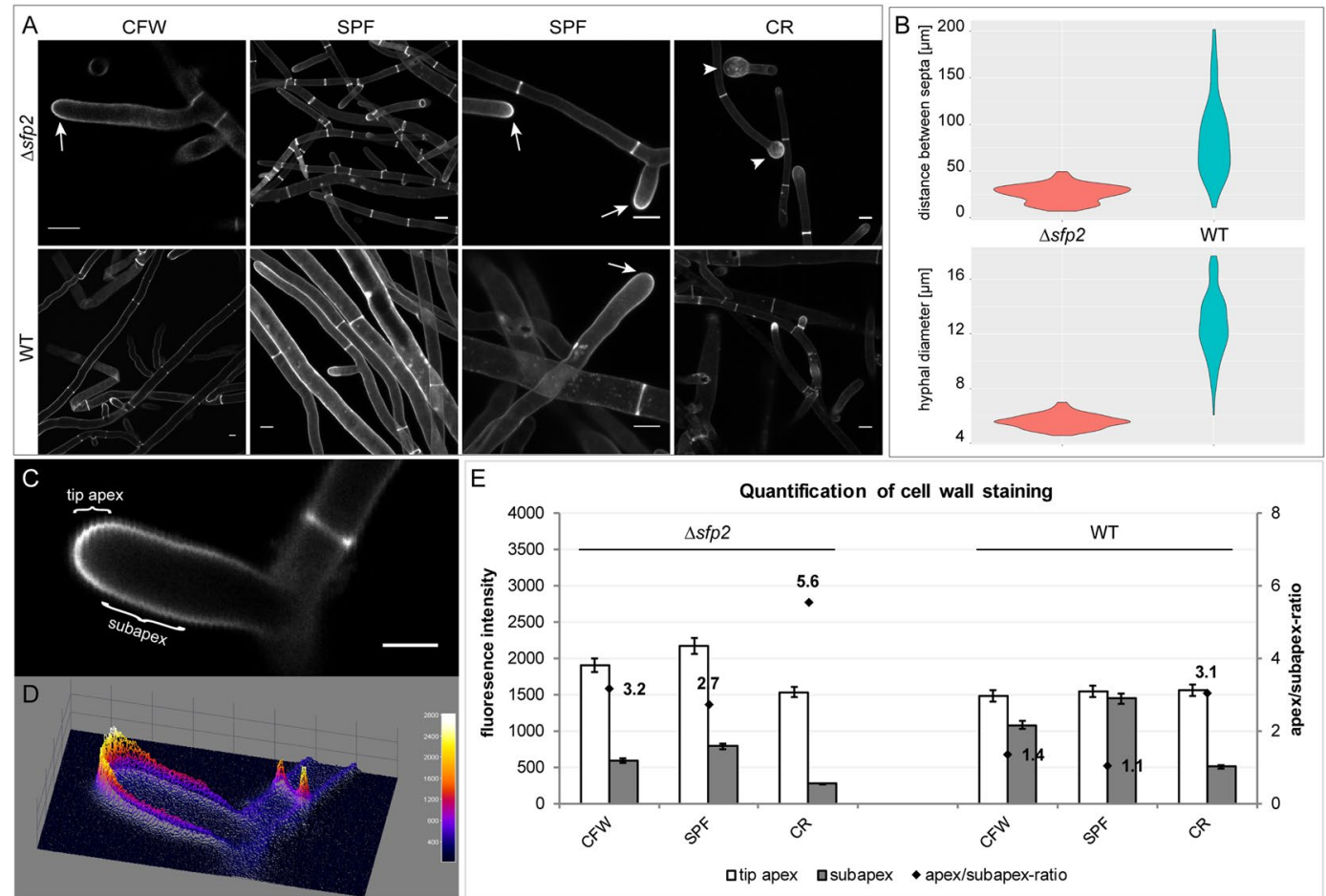

Figure 5. Deletion of $s f p 2$ changes the deposition pattern of cell wall material and affects hyphal morphogenesis (A) Calcofluor White (CFW) and Solophenyl Flavine (SPF) are non-specific stains of $\beta$-1,4-glucans, including chitin, and indicate that more cell wall polymers are deposited at the tip apices of $\Delta s f p 2$ hyphae (arrows) compared to the WT. Congo Red (CR), regarded as a specific stain of $\alpha$ - and $\beta$-chitins, disrupts polarized tip extension much more effectively in $\Delta s f p 2$ than in the WT, leading to extensive tip swelling of mutant hyphae (arrowheads). Scale bars, $10 \mu \mathrm{m}$. (B) The morphogenetic defects caused by sfp 2 deletion become obvious by the significantly reduced average distance between septa $\left(\Delta s f p 2=26.0 \mu \mathrm{m}, \mathrm{WT}=85 \mu \mathrm{m} ; \mathrm{n}=60\right.$; ANOVA $\left.\operatorname{Pr}<2^{-16}\right)$ and the much smaller average hyphal diameter $\left(\Delta s f p 2=5.6 \mu \mathrm{m}\right.$, WT $=12.6 \mu \mathrm{m} ; \mathrm{n}=100$; ANOVA $\left.\operatorname{Pr}<2^{-16}\right)$. (C) Imaging example showing increased dye fluorescence in the tip apex compared to the subapical cell wall region. Scale bar, $5 \mu \mathrm{m}$. (D) 3D surface plot of (C) showing the relative distribution of dye incorporation as colour-coded intensity map. (E) Quantification of the relative fluorescence intensities of cell wall staining in $\Delta s f p 2$ mutant compared to the WT $(\mathrm{n}=55)$.

\section{Discussion}

Mycoparasitism is a trait prominent within the genus Trichoderma. Signaling via heterotrimeric G proteins plays a major role in regulating mycoparasitism-related functions ${ }^{4}$ and in T. atroviride, the seven-transmembrane receptor Gpr1 was found to be essential for the antagonistic interaction of the mycoparasite with prey fungi ${ }^{9}$ as well as for vegetative growth, conidiation and conidial germination ${ }^{7}$. The gpr1-si mutants were unable to respond to the presence of a living prey fungus; consequently, prey hyphae remained intact and undamaged upon confrontation with the mutants 9 . We compared the gene expression response of the gprl-si mutant and its parental strain upon contact with the prey $R$. solani. The top ten genes that were most robustly identified as differently regulated encode proteins involved in protein synthesis and cell detoxification (Table S1), including a putative RTA1-like protein whose family comprises fungal proteins with multiple transmembrane regions that may be involved in the efflux of xenobiotics ${ }^{37}$; a putative mitochondrial endoribonuclease of the isochorismatase superfamily presumably involved in processing and turnover of mitochondrial RNAs; translation elongation factor 2; a putative monooxygenase involved in ubiquinone biosynthesis; a glutathione S-transferase catalyzing the conjugation of the reduced form of glutathione to xenobiotic substrates for the purpose of detoxification ${ }^{38}$; a GH3 family protein; a mitochondrial/plastidial beta-ketoacyl-carrier protein reductase; and two proteins with unknown function. Beta-ketoacyl-ACP reductase is a highly conserved enzyme catalyzing the second step of the mitochondrial fatty acid synthesis pathway. The importance of this pathway has recently been recognized in humans, as it is related to lipoic acid synthesis diseases such as mitochondrial respirational dysfunction and accelerated aging ${ }^{39}$. Furthermore, the response of the plant-pathogenic prey to Trichoderma attack likely results in secretion of secondary metabolites and reactive oxygen species (ROS) that activate Trichoderma genes involved in stress response and detoxification such as reductases and MFS transporters. Several such mycoparasitism-relevant genes were differently regulated in the prey responses of WT and mutants, including aspartyl proteases, protein kinases, nonribosomal peptide synthases (NRPSs), methyl- and acetyltransferases, heat shock proteins (HSP), glutathione S-transferases, glutathione synthetase, small secreted cysteine rich proteins (SSCRP), chitinases (GH18), glycoside hydrolase family 16 (GH16) proteins, N-acetylglucosamine transferases, MFS superfamily members, and 

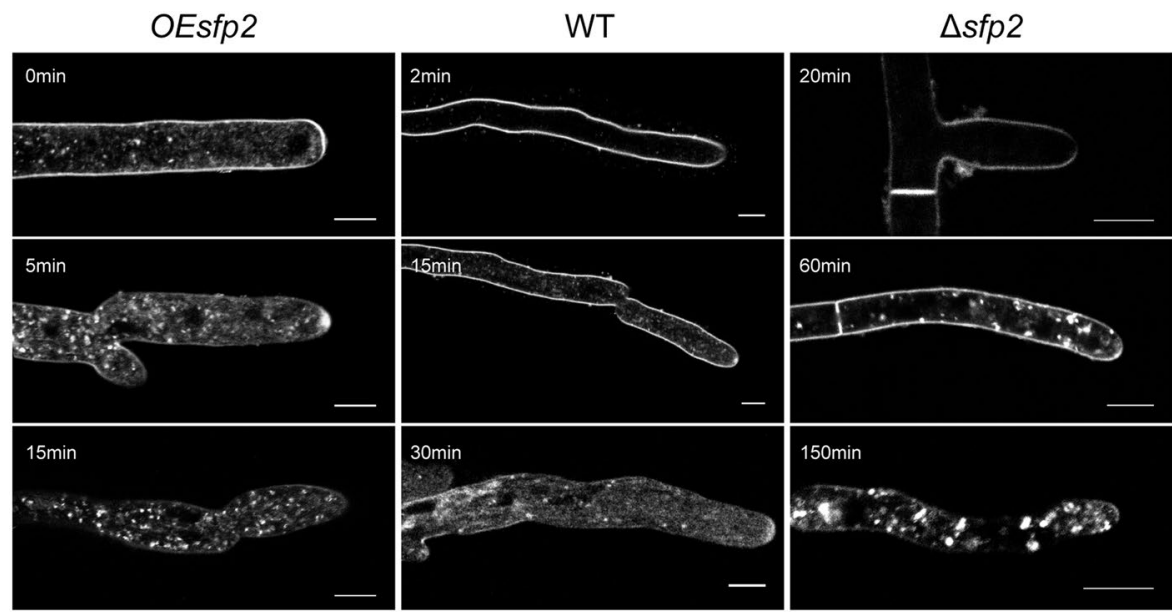

stage I:

plasma membrane

staining

stage II:

appearance of

endocytic vesicles

FM4-64 uptake speed

Figure 6. Influence of Sfp2 on the endocytic uptake of FM4-64. The figure shows three successive key stages of the dye uptake process that are easily discernible in the WT. Stage I: exclusive plasma membrane staining (top row), stage II: first appearance of the dye in endocytic vesicles (middle row), and stage III: exclusive staining of endocytic vesicles and endomembranes (bottom row). Equivalent staining patterns are shown at the earliest time point of their appearance in the respective strain. In comparison to the wild type (WT), endocytosis is slightly accelerated in the $s f p 2$ over-expressing mutant (OEsfp2), whereas dye uptake is dramatically delayed in the gene deletion mutant $(\Delta s f p 2)$. For instance, dye uptake into the plasma membrane occurs instantly in OEsfp2 but takes two minutes in the WT; and complete internalisation of FM4-64 dye from the plasma membrane occurs 10x faster in OEsfp2 compared to $\Delta s f p 2$. Scale bars, $5 \mu \mathrm{m}$.

carbon binding module 50/LysM domain (CBM50) proteins. These results are in accordance with the current model of mycoparasitism where Trichoderma spp. recognize a prey fungus via small molecules (e.g. peptides) that are released from the prey upon the action of secreted Trichoderma hydrolases ${ }^{5}$. The released molecules may bind to Trichoderma cell surface receptors such as Gpr1, thereby eliciting a signaling cascade comprising $G$ proteins, the cAMP pathway and mitogen-activated protein kinases (MAPKs), which may ultimately modulate the activities of transcription factors and consequently the expression of genes that encode enzymes for the biosynthesis of secondary metabolites and cell wall lysis ${ }^{5}$. The transcriptional response to prey of the candidate genes identified in our study depends on Gpr1, including the Tex 1 NRPS, the Tmk2 and Tmk3 MAP kinases and the Lae1 methyltransferase. In T. virens, disruption of the tex 1 gene resulted in the loss of production of 18-residue peptaibols ${ }^{40}$, linear peptide antibiotics produced by Trichoderma and other fungal mycoparasites ${ }^{41}$. Peptaibols directly contribute to the antagonistic action of Trichoderma mycoparasites as they synergistically act with cell wall-degrading enzymes leading to a combined enzymatic degradation of the prey cell wall and membrane permeabilization ${ }^{42,43}$. The Tmk3 MAPK is part of the stress-activated protein kinase (SAPK) signaling pathway and was recently shown to be involved in regulation of osmotic and oxidative stress, cell wall damage, high temperature, cadmium, and UV irradiation in T. atroviride ${ }^{44}$. Tmk3 was rapidly phosphorylated upon light exposure and Tmk3 signaling was suggested to cooperate with the Blr photoreceptor complex in the activation of gene expression $^{44}$. However, in T. atroviride blue-light is perceived through the Blue Light Regulator Complex, which in turn up-regulates a set of genes $(b l u)$ and down-regulates another set $(b l d)$, triggering asexual reproduction ${ }^{27}$. One of the genes, blu7, encoding a $\mathrm{C} 2 \mathrm{H} 2$ zinc finger domain transcription factor, was found to be upregulated in the response of the gpr1-si mutant to R. solani, indicating that blu7 expression is suppressed by Gpr 1 in $T$. atroviride during prey contact. Indeed, Cetz-Chel et al. ${ }^{27}$ suggested that the diminished conidiation of $\Delta b l u 7$ mutants is likely a result of dysregulation of the cAMP signaling pathway and ROS production, whereas their low tolerance to continuous exposure to light indicates that Blu7 is required for adaptation supporting growth under continuous light exposure.

Among the four transmembrane protein-encoding genes differently regulated in the responses of the WT and the gpr1-mutant to R. solani, most of the candidates were putatively engaged in vesicle-mediated transport. This includes the Sur7 proteins, a protein with similarity to Sft2, and a putative orthologue of Der1, a component of the endoplasmic reticulum-associated degradation pathway. The Sft2 proteins might be required for the fusion of transport vesicles derived from the endocytic pathway with the Golgi complex ${ }^{45}$, whereas Sur7 proteins are localized at eisosomes, sites of protein and lipid endocytosis in yeast ${ }^{15}$. Eisosomes, at least partially, mediate this lateral plasma membrane compartmentalization, whereas Sur7-containing MCC are as well important for sphingolipid homeostasis and cell wall morphogenesis ${ }^{11,14,15}$. Little is known about the function of Sur7 family proteins in filamentous fungi. There are several Sur7-like proteins in Aspergillus species, including one strict Sur7 orthologue (SurG in A. nidulans) ${ }^{23,46}$. Vangelatos et al. ${ }^{23}$ showed that in A. nidulans conidiospores, but not in hyphae, SurG and two Pil proteins colocalize at the cell cortex and assemble into eisosomes late during conidial maturation. In mycelia, SurG was found to be located in vacuoles and endosomes. Deletion of the surG gene, however, did not 

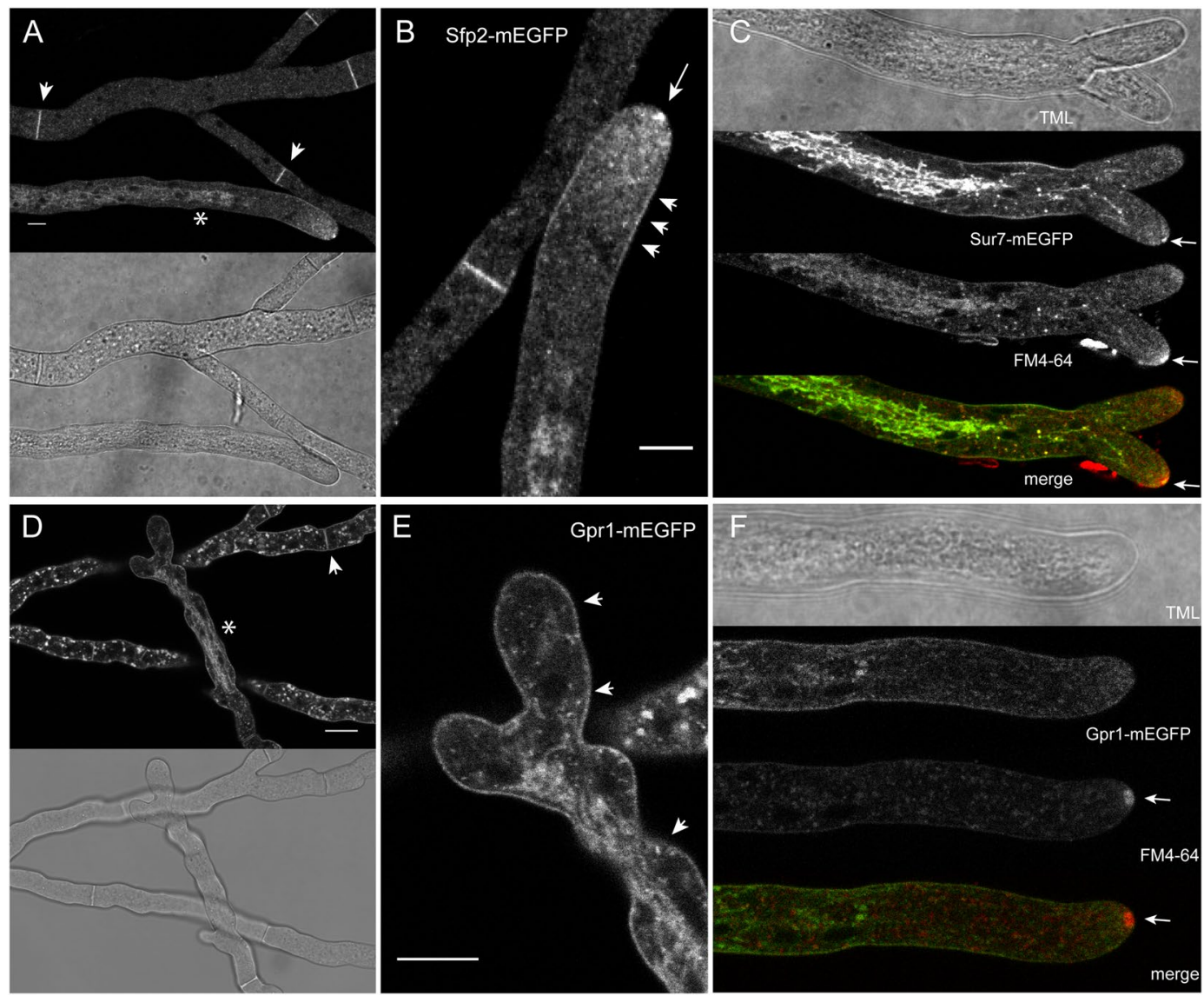

Figure 7. Sfp2-EGFP and Gpr1-EGFP show partially overlapping but not identical subcellular localizations associated to the exo- and endocytic pathways. (A) Sfp2-mEGFP decorates the plasma membrane, septa (arrowheads), and tubular vacuoles (asterisks). (B) Magnification of hyphal tip from (A) showing that besides the plasma membrane (arrowheads), Sfp2-mEGFP is also part of the Spitzenkörper (arrow) and intensely clusters at the position of the subapical endocytic ring (arrowheads). (C) Co-staining with FM4-64 proves that Sfp2 is part of the Spitzenkörper. (D) Gpr1-mEGFP as well localizes to the plasma membrane, septa (arrowheads), and numerous internal membrane systems suspected to include tubular vacuoles (asterisks) and additional compartments, most probably part of the endocytic pathway, that are not obviously occupied by Sfp 2 . (E) Magnification of the hyphal tip from (D) highlighting widespread plasma membrane decoration of Gpr1mEGFP essentially covering the whole hyphal surface (arrowheads). (F) Co-staining with FM4-64 proves that Gpr1 is not part of the Spitzenkörper. Scale bars, $10 \mu \mathrm{m}$.

lead to any obvious growth phenotype, except for moderate resistance to itraconazole. The authors suggested that conservation of eisosomal proteins within the ascomycetes is accompanied by a striking functional divergence.

In this study, we found that the gene encoding the T. atroviride Sur7 family protein Sfp2 is upregulated during the mycoparasitic interaction in a Gpr1-dependent manner. Interestingly, sfp2 and gpr1 are located in spatial proximity in the Trichoderma genomes analyzed and the surrounding genes show synteny, which may be older than the Trichoderma common ancestor, whereas it might not necessarily be older than the Sordariomycetes, as Neurospora crassa (Sordariales) bears no synteny in that region but carries $g p r 1$ and $s f p 2$ on the same supercontig. In the evolutionary distant $A$. nidulans (Eurotiomycetes), moreover, $g p r 1$ and $s f p 2$ orthologues are not part of the same chromosome.

Phylogenetic analysis of Sur7 family proteins from S. cerevisiae, A. nidulans, and diverse Trichoderma species revealed four clades comprising Trichoderma Sur7 family members. One clade gathered orthologues of S. cerevisiae "true" Sur7 and A. nidulans SurG whereas the other three are closely related to S. cerevisiae Sur7 family protein Pun1. As mentioned above, yeast cells lacking the MCC-localized Sur7 protein display broad defects in cellular organization and stress response ${ }^{47}$. We speculate that the reason for the functional discrepancy between yeasts and A. nidulans ${ }^{23}$ might lie in two other S. cerevisiae Sur7 paralogs, Fmp45 and YNL194C, that are more closely related to Trichoderma and A. nidulans SurG than the "true" ScSur7. Pun1, a previously uncharacterized MCC protein, was shown to be nitrogen-stress related, and its absence abolished filamentous growth in haploids and diploids ${ }^{24}$. Though Pun 1 contributes to the cellular response to nitrogen stress through signaling pathways that regulate the expression of genes involved in amino acid biosynthesis ${ }^{24}$, T. atroviride $s f p 2$ deletion mutants did not show significantly altered biomass production upon nitrogen deprivation (data not shown). However, here 
we show that $s f p 2$ deletion results in reduced cell wall stability, increased sensitivity to osmotic and salt stress, and loss of antagonism in the mycoparasite T. atroviride.

Sur7 is needed for proper localization of actin, morphogenesis, cell wall synthesis, and the adequate response to cell wall stress in S. cerevisiae ${ }^{47}$. Similarly, cell wall intercalation by Congo Red strongly reduced growth of the T. atroviride $\Delta s f p 2$ mutant, whereas the WT was not significantly affected. A massive overexpression of subgroup $\mathrm{C}$ chitinase-encoding gene $t a c 2$, responsible for hyphal network formation in T. atroviride, was further observed in the $\Delta s f p 2$ mutant upon contact with its own hyphae, but not when grown in liquid culture. A similar result was obtained for the endochitinase-encoding gene ech 42 during self-confrontation, but also upon contact with $R$. solani. In accordance, ech42 was found to be induced in T. atroviride during growth on colloidal and fungal chitin $^{31}$ and during mycoparasitism ${ }^{48}$. There is also a strong evidence that Ech 42 is involved in autolysis processes in T. atroviride ${ }^{49}$. Moreover, the upregulation of two chitin synthases in the $\Delta s f p 2$ mutant during mycoparasitism further underlines the activation of cell wall repair mechanisms. We also detected an increased expression of actin upon $s f p 2$ deletion. Actin plays an important role in morphogenesis, cytokinesis and the movement of organelles in fungal cells ${ }^{30}$. Many of these processes are mediated by extensive and intimate interactions of actin with cellular membranes ${ }^{50}$. In C. albicans, lack of sur 7 resulted in mislocalization of actin and septin, and abnormal cell wall material protruding into and forming structures within the cytoplasm ${ }^{17,51}$. Our cell wall staining results support the expression data and suggest pronounced apical chitin accumulation at the most actively secreting region of the fungal hyphae, the hyphal tip, whereas chitin consolidation in the most actively endocytosing region - the subapical endocytic ring ${ }^{52,53}$ - was reduced in the $s f p 2$ deletion strain. This correlates with our FM4-64 co-staining results and the dramatically delayed endocytosis rate in that mutant (further details below). Increased deposition of chitin at hyphal tips may as well be associated with the observed lack of hyphal differentiation into leading hyphae plus primary and secondary lateral branches, as well as the significantly decreased distance between septa and the reduced diameter of $\Delta s f p 2$ hyphae as observed in this study. Both, the $\Delta s f p 2$ and $g p r 1$-si mutants form compact colonies with increased lateral branching and significantly less aerial hyphae when compared to the WT and, similar to C. albicans sur $7 \Delta$ mutant $^{51}, \Delta s f p 2$ exhibits intensive invasive growth with hyphae entering the solid medium. The $\Delta s f p 2$ mutant also displayed delayed conidial germination, restricted hyphal elongation and shorter germination tubes compared to the WT and the OEsfp2 strain. This set of features is a typical indicator for a severe polarity defect caused by $s f p 2$ deletion. C. albicans sur $7 \Delta$ mutants exhibited alterations of plasma membrane and cell wall organization by producing irregularly shaped hyphae with obvious intracellular invaginations $s^{54}$. The C. albicans mutants also accumulated cell wall abnormalities over time, thereby indicating defects in spatial and temporal regulation of cell wall synthesis ${ }^{51}$.

Micro- or nanodomain structures on the cell membrane are likely involved in receptor organization and internalization, and consequently influence GPCR signaling. The uniform distribution within the plasma membrane seems unlikely to provide sufficient enrichment to achieve the rapid responses characteristic for GPCRs. Consequently, cells probably concentrate signaling molecules in membrane microdomain ${ }^{55}$, regions of elevated cholesterol and glycosphingolipid content and less fluidity ${ }^{56-58}$. Life-cell imaging analyses of Gpr1 and Sfp2 revealed similar, partly overlapping but not identical, subcellular localization patterns. As expected, both proteins reside in or at the plasma membrane including intensive accumulation at septa, and are associated to intracellular membrane clusters and vesicles, presumably associated to the endocytic pathway as evidenced by FM4-64 co-staining. Both proteins furthermore localize to what appears to be tubular vacuoles in the subapical compartment of leading hyphae. In contrast to mitochondria, these are often densely packed in the apical compartment, which also appear as tubes or filaments ${ }^{59}$. Sfp2 is, in contrast to Gpr1, also part of the Spitzenkörper and appears to form a distinct subapical endocytic ring, which furthermore corroborates its likely function in the exo-/endocytic machinery driving polarized tip growth. This finding is in accordance with the polarized tip growth and endocytosis defect observed in the $s f p 2$ deletion strain. Given the phenotypes described here and the interplay between Gpr1 and Sfp2, we are further investigating whether there is a direct interaction between Gpr1 and Sfp2, and how both proteins dynamically localize in the T. atroviride plasma membrane during mycoparasitism.

\section{Methods}

Strains and culture conditions. T. atroviride strain P1 (ATCC 74058) was used in this study referred to as the wild type (WT). All fungal mutants were derived from this strain including the gprl-silenced mutant gprl sil$8^{9}$. Confrontation assays were performed using Rhizoctonia solani (teleomorph Thanatephorus, Basidiomycota) as a fungal prey. Fungal strains were cultivated and maintained on potato dextrose agar (PDA, Sigma) at $25^{\circ} \mathrm{C}$ in darkness unless otherwise stated. Escherichia coli strains JM109 and Stellar (Clontech, TaKaRa) were used for plasmid constructions and amplification. $20 \mathrm{~mm}^{2}$ mycelial plugs were inoculated on PDA supplemented with $1 \mathrm{M}$ $\mathrm{NaCl}$ and $50 \mathrm{mM}$ sorbitol for analyses of osmotic stress resistance and $200 \mu \mathrm{M}$ Congo Red to test the response to cell wall intercalation. At least three biological replicates per strain were incubated at $25^{\circ} \mathrm{C}$ in darkness and culture development was measured daily. The average growth yield (delta growth) was calculated by subtracting the average growth of the strain cultivated with the stress inducer from the average growth of the strain under control conditions (PDA only). For gene expression analyses, T. atroviride mycelium from dual confrontation assays against itself or against $R$. solani was sampled upon fungal contact. For evaluation of gene expression during cell wall intercalation, PDB (potato dextrose broth, Sigma) with and without $5 \mu \mathrm{g} / \mathrm{ml}$ Congo Red was inoculated with mycelial plugs and incubated for five days at $150 \mathrm{rpm}$ and $25^{\circ} \mathrm{C}$ in darkness.

For transcriptome analyses, confrontation assays of T. atroviride WT and its gprl- silenced mutant against $R$. solani or against itself (control) were performed on PDA plates covered with a cellophane membrane. Plates were incubated at $25^{\circ} \mathrm{C}$ until the mycelia of both fungi made first contact. Peripheral hyphal zones of T. atroviride from at least six independent plates were sampled and shock frozen in liquid nitrogen. The universal reference sample was obtained by growing T. atroviride WT under different conditions (PDA and PDB; liquid synthetic media containing $1 \%$ glycerol, $1 \% \mathrm{~N}$-acetylglucosamine, $1 \%$ chitin, $1 \%$ glucose, or $1 \%$ lactose as previously described ${ }^{60}$; 
in confrontation with $R$. solani) for 24,48 and 72 hours and pooling equal amounts of isolated total RNA. Total RNA was isolated using the PeqGOLD TriFast DNA/RNA/Protein Purification Reagent (PEQLAB Biotechnology, VWR) followed by a further purification step with the RNeasy MiniElute Cleanup Kit (Qiagen, Valencia, CA, USA). The integrity of RNA samples was checked using an Agilent 2100 Bioanalyzer (Agilent, Santa Clara, CA, USA) and a Nanodrop ND-1000 spectrophotometer (Nano-Drop, Wilmington, Germany).

Microarray data analysis. We exploited a custom high-density microarray platform for genome-wide transcriptional profiling of the 11,863 genes listed in the Gene Catalogue version 2 of the T. atroviride genome database. Probes were designed to be specific ${ }^{61}$ using the full T. atroviride genomic sequence [http://genome.jgi.doe. gov/Triat2/Triat2.info.html] (accessed June 2017), while also using all published sequences from the prey fungus $R$. solani as antagonistic templates to avoid unwanted cross-talk [https://genome.jgi.doe.gov/Rhiso1/Rhiso1. home.html].

Labeling and hybridization were performed with the Two-color Microarray-Based Gene Expression Analysis-Quick Amp Labelling with Tecan HS Pro Hybridization protocol (V 5.7, May 2008, Agilent Santa Clara, CA). In brief, double-stranded cDNA was synthesized from $50 \mathrm{ng}$ of total RNA using a T7-oligo(dT) primer, followed by in vitro transcription by T7 RNA polymerase with incorporation of Cy3- and Cy5-labeled CTP. $825 \mathrm{ng}$ of the universal reference and confrontation samples labeled once with each of two fluorescent dyes were pooled and fragmented at $60^{\circ} \mathrm{C}$ for 30 minutes in a $30 \mu \mathrm{l}$ reaction following the manufacturers' instructions. $30 \mu \mathrm{l}$ of $2 \mathrm{x}$ GEx Agilent hybridization buffer HI-RPM (Gene expression hybridization kit, Agilent \# 5188-5242) was then added to the fragmentation mixture and hybridized to Agilent SurePrint Custom GE $4 \times 180$ microarrays (Agilent \#G4862A-037207) for 17 hours at $67^{\circ} \mathrm{C}$ in a Tecan HS 4800 Pro Hybridization Station (Tecan, Männedorf, Switzerland) ${ }^{62}$. Each confrontation sample was hybridized against the universal reference sample in four replicates. In total, 16 microarrays were used in dye swap configuration to minimize dye bias. After hybridization, microarrays were washed 1 minute at room temperature with GE Wash Buffer 1 (Agilent \# 5188-5325) and 1 minute with GE Agilent Wash buffer 2 (Agilent \# 5188-5326). Microarrays were scanned with an Agilent G2505C scanner at a resolution of $5 \mu \mathrm{m}$ in double pass mode, with both green and red channels at $100 \%$ laser power, to yield 20 bit tiff images. The scanned images were analyzed with Feature Extraction Software version 10.10.1.1 (Agilent) using default parameters.

All analyses were performed using the statistical environment $\mathrm{R}$ (www.r-project.org) and Bioconductor libraries (www.bioconductor.org). Differential expression calls were made using an empirical Bayes regularized $t$-test in the Bioconductor limma framework after conservative Benjamini-Yekutieli correction for multiple testing for strong control of the false discovery rate (FDR) to $q<5 \%$.

Gpr1-dependent mycoparasitism-relevant genes were identified as follows: First, the strain specific prey responses were assessed by comparing gene expression induced by the prey with gene expression levels under non-mycoparasitic conditions (self-confrontation control). Specifically, we determined the T. atroviride WT strain response (WT-resp = "WT + prey" versus "WT + WT") as well as the gpr1 mutant response $(g p r 1$-resp $=$ "gpr1 + prey" versus "gpr $1+g p r 1 ")$. Subsequently, the WT and mutant responses were compared resulting in a set of genes dependent on Gpr1 under mycoparasitic conditions (WT-resp versus gpr1-resp). This second-level comparison was directly computed as a specific contrast in the linear model, allowing statistical tests of significances to combine evidence across samples.

These tests were both performed for gene transcripts and for functional groups assigned by the FunCat algorithm ${ }^{63}$ employing a Bayesian approach that takes the hierarchical structure of the FunCat categories into account ${ }^{64}$. Improving on traditional tests ${ }^{65}$, the Bayesian approach mitigates issues of noise for Gene Ontology nodes that map to a small numbers of genes, allowing a transfer of knowledge from parent to children nodes in the Gene Ontology graph.

In order to maximize sensitive recall of relevant candidate genes that are robust under method choice, we combined evidence from a range of conservative and more aggressive normalization approaches in rank-product meta-analysis. In line with practice in the field and observations in large-scale benchmark studies ${ }^{66,67}$, we here focus on candidate genes with a large average effect strength, employing a conservative threshold of $\left|\operatorname{aLog}_{2} \mathrm{FC}\right|>1.5$.

Based on database gene annotation, every gene implicated in the transcriptome analysis was manually curated, and Pfam and InterPro functional protein domains were identified using the NCBI Basic Local Alignment Search Tool (BLAST) for proteins (https://blast.ncbi.nlm.nih.gov/Blast.cgi) and InterproScan sequence search (https:// www.ebi.ac.uk/interpro/search/sequence-search), employing the online tools current at the time with default parameters (September 1, 2016). Prediction of transmembrane helices and signal peptide cleavage sites was performed using TMHMM Server v. $2.0^{68}$ and SignalP $4.1^{69}$ with default parameters as hosted at CBS at the time (December 14, 2016). Table S1 describes the differently regulated genes. Probe level measurement data are available on request and expression profiles are under submission to Array Express.

Growth rate, germination and dual confrontation assays. For assessing the growth rate of T. atroviride WT and mutants, the strains were cultivated on PDA plates at $25^{\circ} \mathrm{C}$ in darkness. Radial growth was measured every 24 hours for 10 days. For the analysis of conidial germination, $100 \mathrm{ml}$ Erlenmeyer flasks containing either $50 \mathrm{ml}$ of PDB or minimal synthetic medium ( $\mathrm{pH} 5.5,2 \mathrm{~g} / \mathrm{L} \mathrm{KH}_{2} \mathrm{PO}_{4}, 1.4 \mathrm{~g} / \mathrm{L}\left(\mathrm{NH}_{4}\right) \mathrm{SO}_{4}, 0.3 \mathrm{~g} / \mathrm{L} \mathrm{CaCl} \mathrm{Cl}_{2} \times$ $2 \mathrm{H}_{2} \mathrm{O}, 0.3 \mathrm{~g} / \mathrm{L} \mathrm{MgSO}_{4} \times 7 \mathrm{H}_{2} \mathrm{O}, 0.05 \%$ peptone, $1 \%$ glucose, $0.5 \mathrm{~g} / \mathrm{L} \mathrm{FeSO}_{4} \times 7 \mathrm{H}_{2} \mathrm{O}, 0.2 \mathrm{~g} / \mathrm{L} \mathrm{ZnSO} \mathrm{Zn}_{4} \times 7 \mathrm{H}_{2} \mathrm{O}, 0.2 \mathrm{~g} / \mathrm{L}$ $\mathrm{MnSO}_{4} \times 7 \mathrm{H}_{2} \mathrm{O}$ ) were inoculated with $10^{5}$ spores $/ \mathrm{ml}$ medium (final concentration). After 12 hours of incubation at $25^{\circ} \mathrm{C}$ and $200 \mathrm{rpm}$, conidial germination was investigated using a Thoma chamber $(0.100 \mathrm{~mm}$ depth Profondeur, $0.0025 \mathrm{~mm}^{2}$ ) and a Nikon optiphot-2 microscope.

Confrontation assays of T. atroviride against $R$. solani were performed as described above. Plates were incubated for seven days and the growth of Trichoderma against itself was set up as a zero inhibition rate. The growth 
inhibition of R. solani was calculated implying that the distance between the plugs of both fungi is $100 \%$. Growth inhibition of the prey fungus was calculated as a percentage of Trichoderma growth corrected for the growth against itself.

Plasmid constructions and genetic manipulation of $T$. atroviride. T. atroviride gene deletion strains were created by the split marker technique using the E. coli hph gene under control of the A. nidulans gpd1 promoter and $\operatorname{trpC}$ terminator as selection marker ${ }^{60}$. All rounds of PCR amplification and fusion reactions were performed by double joint-PCR ${ }^{70}$ using primers listed in Table S3. Flanking regions ( $1500 \mathrm{bp}$ upstream and downstream of the $s f p 2$ coding region) were amplified from $T$. atroviride genomic DNA using primers promoter sur7fw/sur7 Rv (upstream) and terminator sur7fw/surRv (downstream). $h p h$ split marker fragments ${ }^{72}$ were amplified from pBluescript II KS (-)_hph plasmid ${ }^{71} .3 \mu \mathrm{g}$ of each DNA fragment were used for protoplast-mediated fungal transformation. Transformants were purified by three rounds of single spore isolation and deletion of the sfp2 gene (Ta156007) confirmed by PCR genotyping using the primer pair P5Fw/P5Rv (Table S3), located in the flanking regions, outside of the integrated deletion cassette. In order to create $s f p 2$ overexpressing mutants, the gene was amplified from genomic DNA and cloned downstream of the constitutively active T. reesei tef1 promoter (GenBank Accession No. Z23012.1).

For complementation of the $\Delta s f p 2$ mutant, a vector expressing sfp2 C-terminally fused to mEGFP (from pEGFP-N1, GenBank Accession No. U55762.1) and expressed under control of the T. reesei pki1 promoter ${ }^{73}$ was integrated into its genome and transformants selected based on their nourseothricin resistance mediated by the nat1 gene ${ }^{74}$. The Ppki1::sfp2-mEGFP::Tsfp2 construct was also introduced into T. atroviride wild type background. The same approach was used to generate strains expressing Gpr1-mEGFP fusion proteins from a Ppki1: :gpr1-mEGFP::Tgpr1 cassette. All primers used for cloning, transformation and genotypic verification are listed in Table S3. Transformants growing in the presence of $300 \mu \mathrm{g} / \mathrm{ml}$ nourseothricin sulfate (Jena Bioscience) were purified by three rounds of single spore isolation. Genomic integration of the respective expression cassettes was confirmed by genotyping PCR, and expression of fluorescent Sfp2-mEGFP and Gpr1-mEGFP fusion proteins was verified by live-cell imaging microscopy.

Transcript analysis by RT-qPCR. $5 \mu \mathrm{g}$ of DNase I- treated RNA were reverse transcribed with the RevertAid H Minus First Strand cDNA Synthesis Kit (Thermo Scientific) according to the manufacturer's protocol with a combination of the provided oligo $(\mathrm{dT})$ and random hexamer primers. All real-time PCR experiments were performed in triplicates on a Bio-Rad (Hercules, CA) iCycler IQ. $25 \mu \mathrm{l}$ assays with IQ SYBR Green Supermix (Bio-Rad, Hercules, CA), standard $\mathrm{MgCl}_{2}$ concentration $(3 \mathrm{mM}$ ), and a final primer concentration of $100 \mathrm{nM}$ each were used. Primer sequences are provided in Table S4. The amplification protocol consisted of an initial denaturation step $\left(2 \mathrm{~min}\right.$ at $\left.95^{\circ} \mathrm{C}\right)$ followed by 40 cycles of denaturation $\left(5 \mathrm{sec}\right.$ at $\left.95^{\circ} \mathrm{C}\right)$, annealing $(20 \mathrm{sec}$, for Tm see Table S4) and extension $\left(65^{\circ} \mathrm{C}\right.$ for $\left.10 \mathrm{sec}\right)$ followed by a melting curve analysis. Determination of qPCR efficiency was performed using triplicate reactions from a dilution series $\left(1,0.1,10^{-2}\right.$ and $\left.10^{-3}\right)$ of cDNA. Amplification efficiency was calculated from the given slopes in the IQ5 Optical system Software v2.0. Expression ratios were calculated using Livak test model ${ }^{75}$ with sarl as reference gene ${ }^{7}$ and are given in Table S4.

In addition to RT-qPCR analyses tac2, tac6, ech42 (chi18-5) and tef1 listed in Gruber et al. ${ }^{31}$ were used for semi-quantitative RT-PCR. The tef1 gene was employed as reference whereas ech 42 was a positive control for the induction of the chitin degradation machinery. The amplification protocol included an initial denaturation for $30 \mathrm{sec}$ at $98^{\circ} \mathrm{C}$ followed by 26 cycles of denaturation $\left(1 \mathrm{~min}\right.$ at $\left.98^{\circ} \mathrm{C}\right)$, annealing $(1 \mathrm{~min}$, for Tm see Table S4) and extension $\left(72^{\circ} \mathrm{C}\right.$ for $\left.1 \mathrm{~min}\right)$. Final extension was performed for $5 \mathrm{~min}$ at $72^{\circ} \mathrm{C}$.

Phylogenetic analysis. We examined the genomic gene content and position of Trichoderma Sur7 family proteins in the phylogenetic tree based on their sequence similarity to $S$. cerevisiae and A. nidulans Sur7 family members ${ }^{15}$. Protein sequences were obtained by BLAST from each of the Trichoderma spp. and A. nidulans genomes available on the JGI portal (http://genome.jgi.doe.gov), NCBI (https://www.ncbi.nlm.nih.gov/genome/), and from http://www.yeastgenome.org/ for S. cerevisiae. Predicted amino acid sequences were aligned by the MUSCLE analysis tool ${ }^{76}$ and the Dayhoff amino acid substitution model was applied for Bayesian analysis. Metropolis-Coupled Markov Chain Monte Carlo (MCMCMC) sampling was performed using MrBayes v3.2.5 with two simultaneous runs of four incrementally heated chains that performed three million generations in total. Trees were summarized after dropping the first $25 \%$ of the trees (burn-in). Two completely independent analyses starting from different random trees were carried out. Bayesian posterior probabilities (PP) were obtained from the $50 \%$ majority-rule consensus of trees sampled every 100 generations after burn-in. PP values lower than 0.95 were not considered significant and are not shown in the resulting cladogram.

Confocal laser scanning microscopy. Strains expressing either Gpr1-mEGFP or Sfp2-mEGFP were grown on PDA for $48 \mathrm{~h}$ at $25^{\circ} \mathrm{C}$ and $12 \mathrm{~h}$ dark/light cycles. Agar blocks of about $1 \mathrm{~cm}^{2}$ carrying the mycelium were cut out and inverted onto a glass cover slide. Images were recorded on a Nikon C1 confocal laser-scanning unit mounted on a Nikon Eclipse TE2000-E inverted microscope base. EGFP-labeled proteins were excited with the $488 \mathrm{~nm}$ laser line of an argon ion laser, and emitted fluorescence light separated by a Nikon MHX40500b/ C100332 filter cube was detected with a photomultiplier tube within the range of 500-530 nm. A Nikon Plan Apo VC $60 \times / 1.2$ water immersion objective lens was used. Bright-field images were captured simultaneously with a Nikon C1-TD transmitted light detector mounted behind the condenser turret.

Visualization of $\Delta s f p 2$ and WT cell walls was performed on the same Nikon C1 LSCM system using the following cell wall stains: Calcofluor White (CFW) M2R (Sigma \#F3543) at a final concentration of $2 \mu \mathrm{M}$ to non-specifically label $\beta$-1,4-glucans including chitin, Solophenyl Flavine (SPF) 7GFE 500 (Ciba \#1485385V6) at a final concentration of $20 \mu \mathrm{M}$ to selectively label polysaccharides containing $\beta$-1,4 linkages, and Congo Red (CR) 
(Sigma \#C6767) at a final concentration of $100 \mu \mathrm{M}$ to very specifically label $\alpha$ - and $\beta$-chitin. All dyes were left to incubate on the cells for at least $15 \mathrm{~min}$ before imaging. CFW and SPF were excited with $405 \mathrm{~nm}$ light from a blue diode and emission light was detected between $430-470 \mathrm{~nm}$. CR was excited with $543 \mathrm{~nm}$ from a HeNe laser and emission light was detected between 580-620 nm. Measurements of distances between septa and of hyphal diameters were performed with the corresponding plugins of the MacBiophotonics ImageJ work package available at (https://www.macbiophotonics.ca/software.htm), and statistically evaluated using the ggplot2 package of $\mathrm{R}$ (https://www.r-project.org/).

To assess endocytosis in growing hyphae, the membrane-selective fluorescent dye FM4-64 (\#T3166, ThermoFisher Scientific) was used at a final concentration of $1.67 \mu \mathrm{M}$ and imaged on a Leica LSM SP5 confocal microscope fitted with a Leica $63 x / 1.3$ N.A. objective lens. Fluorophore excitation was achieved with the $488 \mathrm{~nm}$ line of an Argon laser, and emission light was detected with a PMT between $600 \mathrm{~nm}$ and $700 \mathrm{~nm}$. For time course recordings, images were taken with a frame rate of $1 \mathrm{~min}^{-1}$ over the desired period for up to $150 \mathrm{~min}$. To evaluate differences in endocytosis rates between the tested strains, we used three key stages of the dye uptake process as readily discernable visual markers (stage I: plasma membrane staining, stage II: appearance of endocytic vesicles and stage III: complete dye internalization) and recorded the time at which these phenotypes first appeared in each strain after dye addition.

In all cases, excitation laser intensity and laser dwell time during image acquisition were kept to a minimum to reduce photobleaching and phototoxic effects while providing a sufficient signal-to-noise ratio for quantitative image analysis. All images were recorded with a maximum resolution of $1024 \times 1024$ pixels and saved as TIFF. Apart from display range adjustments and cropping using the ImageJ software platform (http://rsb.info.nih.gov/ij/), images were not subjected to further manipulation.

Data availability. All data generated or analysed during this study are included in this published article and its Supplementary information files and are available on reasonable request.

\section{References}

1. Vinale, F. et al. Trichoderma-plant-pathogen interactions. Soil Biol. Biochem. 40, 1-10 (2008).

2. Benítez, T., Rincón, A. M., Limón, M. C. \& Codón, A. C. Biocontrol mechanisms of Trichoderma strains. Int. Microbiol. Off. J. Span. Soc. Microbiol. 7, 249-260 (2004).

3. Atanasova, L. Ecophysiology of Trichoderma in Genomic Perspective. In Biotechnology and Biology of Trichoderma $25-40$ (Elsevier). https://doi.org/10.1016/B978-0-444-59576-8.00002-3 (2014)

4. Gruber, S., Omann, M. \& Zeilinger, S. Comparative analysis of the repertoire of G protein-coupled receptors of three species of the fungal genus Trichoderma. BMC Microbiol. 13, 108 (2013).

5. Druzhinina, I. S. et al. Trichoderma: the genomics of opportunistic success. Nat. Rev. Microbiol. 9, 749-759 (2011).

6. Xue, C., Hsueh, Y.-P. \& Heitman, J. Magnificent seven: roles of G protein-coupled receptors in extracellular sensing in fungi. FEMS Microbiol. Rev. 32, 1010-1032 (2008).

7. Brunner, K. et al. Trichoderma G protein-coupled receptors: functional characterisation of a cAMP receptor-like protein from Trichoderma atroviride. Curr. Genet. 54, 283-299 (2008).

8. Schmoll, M. et al. The Genomes of Three Uneven Siblings: Footprints of the Lifestyles of Three Trichoderma Species. Microbiol. Mol. Biol. Rev. 80, 205-327 (2016).

9. Omann, M. R., Lehner, S., Escobar Rodriguez, C., Brunner, K. \& Zeilinger, S. The seven-transmembrane receptor Gpr1 governs processes relevant for the antagonistic interaction of Trichoderma atroviride with its host. Microbiology 158, 107-118 (2012).

10. Malinsky, J., Opekarová, M., Grossmann, G. \& Tanner, W. Membrane microdomains, rafts, and detergent-resistant membranes in plants and fungi. Annu. Rev. Plant Biol. 64, 501-529 (2013).

11. Malinska, K. Visualization of Protein Compartmentation within the Plasma Membrane of Living Yeast Cells. Mol. Biol. Cell 14, 4427-4436 (2003).

12. Malinsky, J., Opekarová, M. \& Tanner, W. The lateral compartmentation of the yeast plasma membrane. Yeast Chichester Engl. 27, 473-478 (2010).

13. Berchtold, D. \& Walther, T. C. TORC2 Plasma Membrane Localization Is Essential for Cell Viability and Restricted to a Distinct Domain. Mol. Biol. Cell 20, 1565-1575 (2009).

14. Walther, T. C. et al. Eisosomes mark static sites of endocytosis. Nature 439, 998-1003 (2006).

15. Douglas, L. M. \& Konopka, J. B. Fungal membrane organization: the eisosome concept. Annu. Rev. Microbiol. 68, 377-393 (2014).

16. Stradalova, V. et al. Furrow-like invaginations of the yeast plasma membrane correspond to membrane compartment of Can1. J. Cell Sci. 122, 2887-2894 (2009).

17. Alvarez, F. J., Douglas, L. M., Rosebrock, A. \& Konopka, J. B. The Sur7 protein regulates plasma membrane organization and prevents intracellular cell wall growth in Candida albicans. Mol. Biol. Cell 19, 5214-5225 (2008).

18. Grossmann, G. et al. Plasma membrane microdomains regulate turnover of transport proteins in yeast. J. Cell Biol. 183, 1075-1088 (2008).

19. Fröhlich, F. et al. A genome-wide screen for genes affecting eisosomes reveals Nce102 function in sphingolipid signaling. J. Cell Biol. 185, 1227-1242 (2009).

20. Young, M. E. et al. The Sur7p family defines novel cortical domains in Saccharomyces cerevisiae, affects sphingolipid metabolism, and is involved in sporulation. Mol. Cell. Biol. 22, 927-934 (2002).

21. Alvarez, F. J., Douglas, L. M. \& Konopka, J. B. The Sur7 protein resides in punctate membrane subdomains and mediates spatial regulation of cell wall synthesis in Candida albicans. Commun. Integr. Biol. 2, 76-77 (2009).

22. Alvarez, F. J. \& Konopka, J. B. Identification of an N-acetylglucosamine transporter that mediates hyphal induction in Candida albicans. Mol. Biol. Cell 18, 965-975 (2007).

23. Vangelatos, I. et al. Eisosome organization in the filamentous ascomycete Aspergillus nidulans. Eukaryot. Cell 9, 1441-1454 (2010).

24. Xu, T. et al. A profile of differentially abundant proteins at the yeast cell periphery during pseudohyphal growth. J. Biol. Chem. 285, 15476-15488 (2010).

25. Hosiner, D. et al. Pun 1p is a metal ion-inducible, calcineurin/Crzlp-regulated plasma membrane protein required for cell wall integrity. Biochim. Biophys. Acta 1808, 1108-1119 (2011).

26. Karimi Aghcheh, R., Druzhinina, I. S. \& Kubicek, C. P. The putative protein methyltransferase LAE1 of Trichoderma atroviride is a key regulator of asexual development and mycoparasitism. PloS One 8, e67144 (2013).

27. Cetz-Chel, J. E., Balcázar-López, E., Esquivel-Naranjo, E. U. \& Herrera-Estrella, A. The Trichoderma atroviride putative transcription factor Blu7 controls light responsiveness and tolerance. BMC Genomics 17, 327 (2016). 
28. Seidl, V., Marchetti, M., Schandl, R., Allmaier, G. \& Kubicek, C. P. Epl1, the major secreted protein of Hypocrea atroviridis on glucose, is a member of a strongly conserved protein family comprising plant defense response elicitors. FEBS J. 273, 4346-4359 (2006).

29. Casas-Flores, S., Rios-Momberg, M., Bibbins, M., Ponce-Noyola, P. \& Herrera-Estrella, A. BLR-1 and BLR-2, key regulatory elements of photoconidiation and mycelial growth in Trichoderma atroviride. Microbiol. Read. Engl. 150, 3561-3569 (2004).

30. Kopecká, M. \& Gabriel, M. The influence of congo red on the cell wall and (3)-beta-D-glucan microfibril biogenesis in Saccharomyces cerevisiae. Arch. Microbiol. 158, 115-126 (1992).

31. Gruber, S. et al. Analysis of subgroup C of fungal chitinases containing chitin-binding and LysM modules in the mycoparasite Trichoderma atroviride. Glycobiology 21, 122-133 (2011).

32. Gruber, S. \& Seidl-Seiboth, V. Self versus non-self: fungal cell wall degradation in Trichoderma. Microbiology 158, 26-34 (2012).

33. Lenardon, M. D., Munro, C. A. \& Gow, N. A. R. Chitin synthesis and fungal pathogenesis. Curr. Opin. Microbiol. 13, 416-423 (2010).

34. Kong, L.-A. et al. Different chitin synthase genes are required for various developmental and plant infection processes in the rice blast fungus Magnaporthe oryzae. PLoS Pathog. 8, e1002526 (2012).

35. Gonçalves, I. R. et al. Genome-wide analyses of chitin synthases identify horizontal gene transfers towards bacteria and allow a robust and unifying classification into fungi. BMC Evol. Biol. 16, 252 (2016).

36. Walker, S. K. \& Garrill, A. Actin microfilaments in fungi. Mycologist 20, 26-31 (2006)

37. Soustre, I., Letourneux, Y. \& Karst, F. Characterization of the Saccharomyces cerevisiae RTA1 gene involved in 7-aminocholesterol resistance. Curr. Genet. 30, 121-125 (1996).

38. Sheehan, D., Meade, G., Foley, V. M. \& Dowd, C. A. Structure, function and evolution of glutathione transferases: implications for classification of non-mammalian members of an ancient enzyme superfamily. Biochem. J. 360, 1 (2001).

39. Venkatesan, R. et al. Insights into mitochondrial fatty acid synthesis from the structure of heterotetrameric 3-ketoacyl-ACP reductase/3R-hydroxyacyl-CoA dehydrogenase. Nat. Commun. 5, 4805 (2014).

40. Viterbo, A., Wiest, A., Brotman, Y., Chet, I. \& Kenerley, C. The 18 mer peptaibols from Trichoderma virens elicit plant defence responses. Mol. Plant Pathol. 8, 737-746 (2007).

41. Wiest, A. et al. Identification of peptaibols from Trichoderma virens and cloning of a peptaibol synthetase. J. Biol. Chem. 277, 20862-20868 (2002).

42. Schirmböck, M. et al. Parallel formation and synergism of hydrolytic enzymes and peptaibol antibiotics, molecular mechanisms involved in the antagonistic action of Trichoderma harzianum against phytopathogenic fungi. Appl. Environ. Microbiol. 60, 4364-4370 (1994)

43. Lorito, M., Farkas, V., Rebuffat, S., Bodo, B. \& Kubicek, C. P. Cell wall synthesis is a major target of mycoparasitic antagonism by Trichoderma harzianum. J. Bacteriol. 178, 6382-6385 (1996).

44. Esquivel-Naranjo, E. U. et al. A Trichoderma atroviride stress-activated MAPK pathway integrates stress and light signals. Mol. Microbiol. 100, 860-876 (2016).

45. Conchon, S., Cao, X., Barlowe, C. \& Pelham, H. R. Got1p and Sft2p: membrane proteins involved in traffic to the Golgi complex. EMBO J. 18, 3934-3946 (1999).

46. Athanasopoulos, A., Gournas, C., Amillis, S. \& Sophianopoulou, V. Characterization of AnNce102 and its role in eisosome stability and sphingolipid biosynthesis. Sci. Rep. 5, 15200 (2015).

47. Douglas, L. M., Wang, H. X., Li, L. \& Konopka, J. B. Membrane Compartment Occupied by Can1 (MCC) and Eisosome Subdomains of the Fungal Plasma Membrane. Membranes 1, 394-411 (2011).

48. Zeilinger, S. et al. Chitinase gene expression during mycoparasitic interaction of Trichoderma harzianum with its host. Fungal Genet. Biol. FG B 26, 131-140 (1999).

49. Carsolio, C., Gutiérrez, A., Jiménez, B., Van Montagu, M. \& Herrera-Estrella, A. Characterization of ech-42, a Trichoderma harzianum endochitinase gene expressed during mycoparasitism. Proc. Natl. Acad. Sci. USA 91, 10903-10907 (1994).

50. Doherty, G. J. \& McMahon, H. T. Mediation, modulation, and consequences of membrane-cytoskeleton interactions. Annu. Rev. Biophys. 37, 65-95 (2008).

51. Wang, H. X., Douglas, L. M., Aimanianda, V., Latge, J.-P. \& Konopka, J. B. The Candida albicans Sur7 Protein Is Needed for Proper Synthesis of the Fibrillar Component of the Cell Wall That Confers Strength. Eukaryot. Cell 10, 72-80 (2011)

52. Berepiki, A., Lichius, A. \& Read, N. D. Actin organization and dynamics in filamentous fungi. Nat. Rev. Microbiol. 9, 876-887 (2011).

53. Riquelme, M. \& Martínez-Núñez, L. Hyphal ontogeny in Neurospora crassa: a model organism for all seasons. F1000Research 5, 2801 (2016).

54. Bernardo, S. M. \& Lee, S. A. Candida albicans SUR7 contributes to secretion, biofilm formation, and macrophage killing. BMC Microbiol. 10, $133(2010)$

55. Ostrom, R. S. \& Insel, P. A. The evolving role of lipid rafts and caveolae in G protein-coupled receptor signaling: implications for molecular pharmacology. Br. J. Pharmacol. 143, 235-245 (2004).

56. Pike, L. J. Lipid rafts: bringing order to chaos. J. Lipid Res. 44, 655-667 (2003).

57. Jacobson, K., Mouritsen, O. G. \& Anderson, R. G. W. Lipid rafts: at a crossroad between cell biology and physics. Nat. Cell Biol. 9 , 7-14 (2007).

58. Foster, L. J., de Hoog, C. L. \& Mann, M. Unbiased quantitative proteomics of lipid rafts reveals high specificity for signaling factors. Proc. Natl. Acad. Sci. 100, 5813-5818 (2003).

59. Bowman, B. J. et al. Characterization of a Novel Prevacuolar Compartment in Neurospora crassa. Eukaryot. Cell 14, 1253-1263 (2015).

60. Gruber, S. \& Zeilinger, S. The Transcription Factor Ste12 Mediates the Regulatory Role of the Tmk1 MAP Kinase in Mycoparasitism and Vegetative Hyphal Fusion in the Filamentous Fungus Trichoderma atroviride. PLoS ONE 9, el11636 (2014).

61. Leparc, G. G. et al. Model-based probe set optimization for high-performance microarrays. Nucleic Acids Res. 37, e18 (2009).

62. Sykacek, P. et al. The impact of quantitative optimization of hybridization conditions on gene expression analysis. BMC Bioinformatics 12, 73 (2011).

63. Ruepp, A. The FunCat, a functional annotation scheme for systematic classification of proteins from whole genomes. Nucleic Acids Res. 32, 5539-5545 (2004)

64. Zhang, S., Cao, J., Kong, Y. M. \& Scheuermann, R. H. GO-Bayes: Gene Ontology-based overrepresentation analysis using a Bayesian approach. Bioinforma. Oxf. Engl. 26, 905-911 (2010).

65. Huang, D. W., Sherman, B. T. \& Lempicki, R. A. Bioinformatics enrichment tools: paths toward the comprehensive functional analysis of large gene lists. Nucleic Acids Res. 37, 1-13 (2009).

66. MAQC Consortium et al. The MicroArray Quality Control (MAQC) project shows inter- and intraplatform reproducibility of gene expression measurements. Nat. Biotechnol. 24, 1151-1161 (2006)

67. SEQC/MAQC-III Consortium. A comprehensive assessment of RNA-seq accuracy, reproducibility and information content by the Sequencing Quality Control Consortium. Nat. Biotechnol. 32, 903-914 (2014).

68. Krogh, A., Larsson, B., von Heijne, G. \& Sonnhammer, E. L. Predicting transmembrane protein topology with a hidden Markov model: application to complete genomes. J. Mol. Biol. 305, 567-580 (2001).

69. Petersen, T. N., Brunak, S., von Heijne, G. \& Nielsen, H. SignalP 4.0: discriminating signal peptides from transmembrane regions. Nat. Methods 8, 785-786 (2011). 
70. Yu, J.-H. et al. Double-joint PCR: a PCR-based molecular tool for gene manipulations in filamentous fungi. Fungal Genet. Biol. FG B 41, 973-981 (2004).

71. Mach, R. L., Schindler, M. \& Kubicek, C. P. Transformation of Trichoderma reesei based on hygromycin B resistance using homologous expression signals. Curr. Genet. 25, 567-570 (1994).

72. Catlett, N. L., Lee, B.-N., Yoder, O. C. \& Turgeon, B. G. Split-Marker Recombination for Efficient Targeted Deletion of FungalGenes. Fungal Genet. Rep. 50, 9-11 (2003).

73. Schindler, M. et al. Characterization of the pyruvate kinase-encoding gene (pki1) of Trichoderma reesei. Gene 130, 271-275 (1993).

74. Kück, U. \& Hoff, B. Application of the nourseothricin acetyltransferase gene (nat1) as dominant marker for the transformation of filamentous fungi. Fungal Genet. Rep. 53, 9-11 (2006).

75. Livak, K. J. \& Schmittgen, T. D. Analysis of Relative Gene Expression Data Using Real-Time Quantitative PCR and the $2^{-\Delta \Delta C T}$ Method. Methods 25, 402-408 (2001).

76. Edgar, R. C. MUSCLE: multiple sequence alignment with high accuracy and high throughput. Nucleic Acids Res. 32, 1792-1797 (2004)

\section{Acknowledgements}

The Vienna Science and Technology Fund WWTF (www.wwtf.at, grant numbers LS09-036 and LS 13-086 to SZ), the Austrian Science Fund FWF (www.fwf.ac.at, grant number V139 to SZ) and the Hungarian Széchenyi 2020 Programme (grant number GINOP-2.3.2-15-2016-00052) are acknowledged for funding. The funders had no role in study design, data collection and analysis, decision to publish, or preparation of the manuscript. LA and SZ are thankful to Prof. Dr. Clemens Peterbauer (University of Natural Resources and Life Sciences, Vienna) for providing labspace to LA, and to Markus Omann and Carolina Escobar for help with cultivations and RNA extraction of samples for microarray analysis. DPK acknowledges HPCF support by the Vienna Scientific Cluster.

\section{Author Contributions}

L.A., S.G., A.L., T.R., L. Ab. and N.S.P. conducted the experiments. L.A., A.L., S.G., D.P.K. and S.Z. designed the study and analyzed the data. L.A. and A.L. prepared the figures; L.A. prepared the supplementary materials. N.S.P. and D.P.K. designed and established the custom transcriptomics platform for T. atroviride, P.P.L., D.P.K., M.M. and L.A. analyzed the transcriptome data. L.A. and S.Z. wrote the initial manuscript. All authors read, revised and approved the manuscript.

\section{Additional Information}

Supplementary information accompanies this paper at https://doi.org/10.1038/s41598-018-30500-y.

Competing Interests: The authors declare no competing interests.

Publisher's note: Springer Nature remains neutral with regard to jurisdictional claims in published maps and institutional affiliations.

Open Access This article is licensed under a Creative Commons Attribution 4.0 International License, which permits use, sharing, adaptation, distribution and reproduction in any medium or format, as long as you give appropriate credit to the original author(s) and the source, provide a link to the Creative Commons license, and indicate if changes were made. The images or other third party material in this article are included in the article's Creative Commons license, unless indicated otherwise in a credit line to the material. If material is not included in the article's Creative Commons license and your intended use is not permitted by statutory regulation or exceeds the permitted use, you will need to obtain permission directly from the copyright holder. To view a copy of this license, visit http://creativecommons.org/licenses/by/4.0/.

(c) The Author(s) 2018 